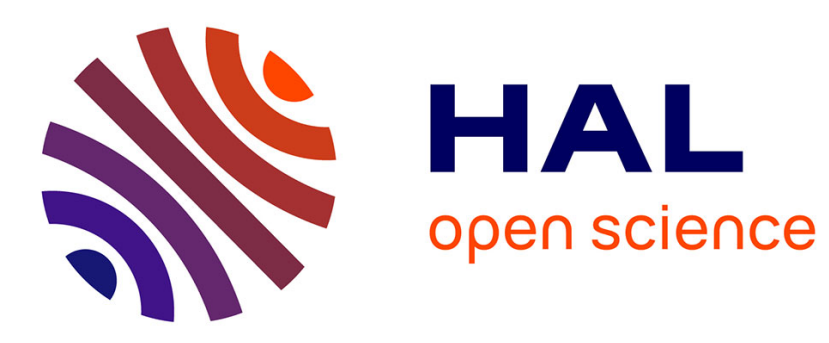

\title{
Two-dimensional thinning algorithms based on critical kernels
}

\author{
Gilles Bertrand, Michel Couprie
}

\section{To cite this version:}

Gilles Bertrand, Michel Couprie. Two-dimensional thinning algorithms based on critical kernels. Journal of Mathematical Imaging and Vision, 2008, 31 (1), pp.35-56. hal-00622001

\section{HAL Id: hal-00622001 \\ https://hal.science/hal-00622001}

Submitted on 11 Sep 2011

HAL is a multi-disciplinary open access archive for the deposit and dissemination of scientific research documents, whether they are published or not. The documents may come from teaching and research institutions in France or abroad, or from public or private research centers.
L'archive ouverte pluridisciplinaire HAL, est destinée au dépôt et à la diffusion de documents scientifiques de niveau recherche, publiés ou non, émanant des établissements d'enseignement et de recherche français ou étrangers, des laboratoires publics ou privés. 


\title{
Two-dimensional parallel thinning algorithms based on critical kernels
}

\author{
G. Bertrand and M. Couprie \\ Institut Gaspard-Monge \\ Laboratoire A2SI, Groupe ESIEE \\ Cité Descartes, BP 99 \\ 93162 Noisy-le-Grand Cedex France
}

\begin{abstract}
Critical kernels constitute a general framework settled in the category of abstract complexes for the study of parallel thinning in any dimension. The most fundamental result in this framework is that, if a subset $Y$ of $X$ contains the critical kernel of $X$, then $Y$ is guaranteed to have "the same topology as $X$ ". Here, we focus on 2D structures in spaces of two and three dimensions. We introduce the notion of crucial pixel which permits to make a link with the framework of digital topology. Thanks to simple local characterizations, we are able to express thinning algorithms by the way of sets of masks. We propose several new parallel algorithms, which are both fast and simple to implement, to obtain symmetrical or non-symmetrical skeletons of $2 \mathrm{D}$ objects in $2 \mathrm{D}$ or $3 \mathrm{D}$ grids. We prove some properties of these skeletons, related to topology preservation, to minimality and to the inclusion of the topological axis which may be seen as a generalization of the medial axis. We also show how to use critical kernels in order to prove very simply the topological soundness of existing thinning schemes. At last, we make clear the link between critical kernels, minimal non-simple sets, and P-simple points.
\end{abstract}

Key words:

Parallel thinning algorithms, skeleton, simple point, collapse, critical kernel, medial axis, digital topology, minimal non-simple set, $\mathrm{P}$-simple point

Email addresses: g.bertrand@esiee.fr (G. Bertrand), m.couprie@esiee.fr (M. Couprie). 


\section{Introduction}

Forty years ago, in 1966, D. Rutovitz proposed an algorithm which is certainly the first parallel thinning algorithm [39]. Since then, many 2D parallel thinning algorithms have been proposed, see in particular $[41,35,1,32,8,15,14,19,11,2,30]$. A fundamental property required for such algorithms is that they do preserve the topology of the original objects. In fact, such a guarantee is not obvious to obtain, even for the $2 \mathrm{D}$ case. C. Ronse introduced the minimal non-simple sets [33] to study the conditions under which points may be removed simultaneously while preserving topology of $2 \mathrm{D}$ objects. This leads to verification methods for the topological soundness of parallel thinning algorithms. Such methods have been proposed for 2D algorithms by C. Ronse [33] and R. Hall [16], they have been developed for the 3D case by T.Y. Kong $[20,21]$ and C.M. Ma [29], as well as for the 4D case by C-J. Gau and T.Y. Kong [12,24]. For the 3D case, G. Bertrand [3] introduced the notion of P-simple point as a verification method but also as a methodology to design parallel thinning algorithms $[4,7,26,27]$.

In [5], one of the authors introduces a general framework for the study of parallel thinning in any dimension in the context of abstract complexes. A new definition of a simple point (a point which may be deleted without changing the topology of the object) is proposed, this definition is based on the collapse operation which is a classical tool in algebraic topology and which guarantees topology preservation. Then, the notions of an essential face and of a core of a face allow to define the critical kernel of an object $X$. The most fundamental result proved in [5] is that, if a subset $Y$ of $X$ contains the critical kernel of $X$, then $Y$ has the same topology as $X$.

This article is the first of a series which develops and exploits the framework of critical kernels, recalled in Sec. 3. Here, we focus on 2D structures in 2D and $3 \mathrm{D}$ spaces. Of course, the important particular case of the $2 \mathrm{D}$ grid receives a special attention, and comparisons with previous works are made. We introduce the notions of crucial faces and pixels (Sec. 4, Sec. 6) which permit to make a link with the framework of digital topology $[34,36,25]$. Thanks to simple local characterizations (Sec. 5), we are able to express thinning algorithms by the way of sets of masks, as in most papers related to parallel thinning. In fact, we were able, in most cases, to reduce this set of masks to a single pattern, leading to algorithms which are both fast and extremely simple to implement. We introduce the formal definition of a minimal symmetric skeleton, which is a well-defined object, and we propose an algorithm to compute it (Sec. 7). We propose several new parallel algorithms to compute curvilinear skeletons (Sec. 8), in which topological and geometrical conditions are clearly separated, unlike in many previous works. 
The quality of a curvilinear skeleton is often assessed by the fact that it contains, approximately or completely, the medial axis of the shape. The medial axis is the set of centers of all maximal balls included in the shape; in [38] A. Rosenfeld and J.L. Pfaltz have proved that, for the city block and the chessboard distances, the medial axis of a shape can be obtained by detecting the local maxima of its distance transform. We introduce the topological axis (Sec. 9), a generalization of the medial axis (which is not defined for the case of two-dimensional structures in discrete $n$-dimensional spaces, $n>2$ ). The topological axis also unveils a deep link between the medial axis and the collapse operation. In 2D, we propose four new parallel algorithms (Sec. 10 and Sec. 11) to compute skeletons which are guaranteed to include the medial axis, and compare them with previously proposed ones in terms of the number of pixels retained in order to ensure topology preservation. Our third algorithm, which is not symmetrical, has the property of producing a result which is minimal, in the sense that any pixel which does not belong to the medial axis is not simple. We extend our algorithms to the $3 \mathrm{D}$ case by proposing a new algorithm to compute minimal symmetric skeletons of 2D objects in 3D grids, and also a new algorithm to compute skeletons of 2D objects in 3D grids which are guaranteed to contain the topological axis (Sec. 12).

We show (Sec. 13) that, thanks to the notions introduced in the paper, it is possible to check the topological soundness of thinning algorithms or thinning schemes. To illustrate this fact, we give new proofs relative to three popular thinning schemes. These proofs are based on very simple arguments and consist in only few lines. The thinning schemes which are considered are the ones proposed by A. Rosenfeld [35], R. Hall. [15], U. Eckhardt and G. Maderlechner [11]. We also report the work made in [10] where 15 parallel thinning algorithms are analyzed with the help of computer. At last (Sec. 14), we show that, with critical kernels, it is possible to recover the notion of a minimal non-simple set as well as the notion of a P-simple point.

\section{Cubical complexes}

In this section, we give some basic definitions for cubical complexes. We consider here only the two-dimensional case. The reader is invited to check that many of the notions introduced in the first sections make sense in arbitrary $n$-dimensional cubical spaces.

If $T$ is a subset of $S$, we write $T \subseteq S$, we also write $T \subset S$ if $T \subseteq S$ and $T \neq S$.

Let $\mathbb{Z}$ be the set of integers. We consider the families of sets $\mathbb{F}_{0}^{1}, \mathbb{F}_{1}^{1}$, such that $\mathbb{F}_{0}^{1}=\{\{a\} \mid a \in \mathbb{Z}\}, \mathbb{F}_{1}^{1}=\{\{a, a+1\} \mid a \in \mathbb{Z}\}$. A subset $f$ of $\mathbb{Z}^{n}, n \geq 2$, which 


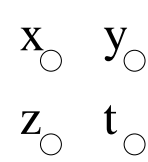

(a)

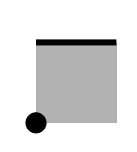

(b)

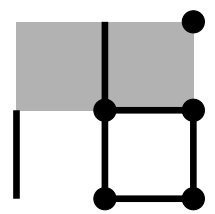

(c)

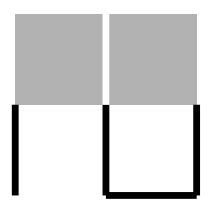

(d)

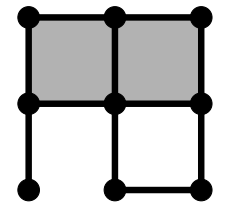

(e)

Fig. 1. (a): Four points $x, y, z, t$. (b): A graphical representation of the set of faces $\{\{x, y, z, t\},\{x, y\},\{z\}\}$ (one 2-face, one 1-face, and one 0-face). (c): A set of faces $X$, which is not a complex. (d): The set $X^{+}$, composed by all the facets of $X$. (e): The set $X^{-}$, i.e. the closure of $X$, which is a complex.

is the Cartesian product of exactly $m$ elements of $\mathbb{F}_{1}^{1}$ and $(n-m)$ elements of $\mathbb{F}_{0}^{1}$ is called a face or an $m$-face of $\mathbb{Z}^{n}, m$ is the dimension of $f$, we write $\operatorname{dim}(f)=m$.

We denote by $\mathbb{F}_{2}^{n}$ the set composed of all $m$-faces of $\mathbb{Z}^{n}, m=0,1,2$ and $n \geq 2$. An $m$-face of $\mathbb{Z}^{n}$ is called a point if $m=0$, a (unit) interval if $m=1$, a (unit) square if $m=2$.

In this paper, we will consider only $2 \mathrm{D}$ objects which are in $2 \mathrm{D}$ or $3 \mathrm{D}$ spaces. Thus, in the following, we suppose that $n=2$ or $n=3$.

Let $f$ be a face in $\mathbb{F}_{2}^{n}$. We set $\hat{f}=\left\{g \in \mathbb{F}_{2}^{n} \mid g \subseteq f\right\}$ and $\hat{f}^{*}=\hat{f} \backslash\{f\}$.

Any $g \in \hat{f}$ is a face of $f$, and any $g \in \hat{f}^{*}$ is a proper face of $f$.

If $X$ is a finite set of faces in $\mathbb{F}_{2}^{n}$, we write $X^{-}=\cup\{\hat{f} \mid f \in X\}, X^{-}$is the closure of $X$.

A set $X$ of faces in $\mathbb{F}_{2}^{n}$ is a cell or an $m$-cell if there exists an $m$-face $f \in X$, such that $X=\hat{f}$. The boundary of a cell $\hat{f}$ is the set $\hat{f}^{*}$.

A finite set $X$ of faces in $\mathbb{F}_{2}^{n}$ is a complex (in $\mathbb{F}_{2}^{n}$ ) if $X=X^{-}$. Any subset $Y$ of a complex $X$ which is also a complex is a subcomplex of $X$. If $Y$ is a subcomplex of $X$, we write $Y \preceq X$. If $X$ is a complex in $\mathbb{F}_{2}^{n}$, we also write $X \preceq \mathbb{F}_{2}^{n}$.

Let $X \preceq \mathbb{F}_{2}^{n}$. A face $f \in X$ is a facet of $X$ if there is no $g \in X$ such that $f \in \hat{g}^{*}$. We denote by $X^{+}$the set composed of all facets of $X$.

Observe that $X^{+}$is, in general, not a complex, and that $\left[X^{+}\right]^{-}=X$.

In Fig. 1, we give some illustrations of the notions defined above for some sets of faces in $\mathbb{F}_{2}^{2}$ (in the "2D square grid").

Let $X \preceq \mathbb{F}_{2}^{n}$. The dimension of $X$ is the number:

$$
\operatorname{dim}(X)=\max \left\{\operatorname{dim}(f) \mid f \in X^{+}\right\} .
$$

We say that $X$ is an $m$-complex if $\operatorname{dim}(X)=m$.

We say that $X$ is pure if, for each $f \in X^{+}$, we have $\operatorname{dim}(f)=\operatorname{dim}(X)$. 


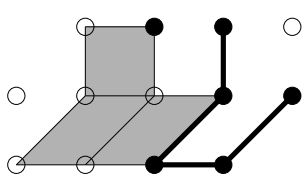

(a)

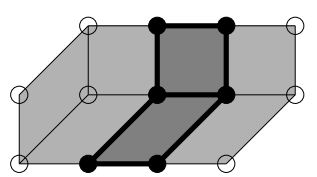

(b)

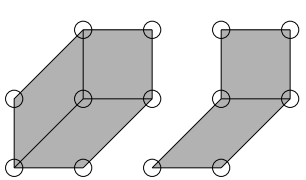

(c)

Fig. 2. (a): A complex $X_{1}$ which is not pure and not connected. Highlighted (bold edges and vertices): a subcomplex $Y_{1}$ of $X_{1}$ which is not a principal subcomplex. (b): A connected, pure 2-complex $X_{2}$. Highlighted: a subcomplex $Y_{2}$ of $X_{2}$ which is a principal subcomplex, and which is also a pure 2-complex. (c): The detachment of $Y_{2}$ from $X_{2}$, a pure 2-complex which is not connected.

Let $X \preceq \mathbb{F}_{2}^{n}$ and $Y \preceq X$. If $Y^{+} \subseteq X^{+}$, we say that $Y$ is a principal subcomplex of $X$ and we write $Y \sqsubseteq X$. Observe that, for any $X \preceq \mathbb{F}_{2}^{n}, \emptyset \sqsubseteq X$.

If $X \preceq \mathbb{F}_{2}^{n}$ and if $X$ is a pure 2-complex, we also write $X \sqsubseteq \mathbb{F}_{2}^{n}$.

Let $X \preceq \mathbb{F}_{2}^{n}$ and let $Y \preceq X$. We set $X \odot Y=\left[X^{+} \backslash Y^{+}\right]^{-}$. The set $X \ominus Y$ is a complex which is the detachment of $Y$ from $X$.

Two distinct faces $f$ and $g$ of $\mathbb{F}_{2}^{n}$ are adjacent if $f \cap g \neq \emptyset$. Two complexes $X$, $Y$ in $\mathbb{F}_{2}^{n}$ are adjacent if there exist $f \in X$ and $g \in Y$ which are adjacent.

Let $X \preceq \mathbb{F}_{2}^{n}$. A sequence $\pi=\left\langle f_{0}, \ldots, f_{l}\right\rangle$ of faces in $X$ is a path in $X$ (from $f_{0}$ to $f_{l}$ ) if $f_{i}$ and $f_{i+1}$ are adjacent for each $i=0, \ldots, l-1$; the number $l$ is the length of $\pi$.

We say that $X$ is connected if, for any pair of faces $(f, g)$ in $X$, there is a path in $X$ from $f$ to $g$. We say that $Y \preceq X$ is a connected component of $X$ if $Y \subseteq X, Y$ is connected, and if $Y$ is maximal for these two properties (i.e., we have $Z=Y$ whenever $Y \preceq Z \preceq X$ and $Z$ connected).

Fig. 2 illustrates the notions of pureness, connectedness, subcomplex, principal subcomplex and detachment for some $2 \mathrm{D}$ complexes in $\mathbb{F}_{2}^{3}$ (in the " $3 \mathrm{D}$ cubic grid").

Two 2-faces $f$ and $g$ of $\mathbb{F}_{2}^{n}$ are strongly adjacent if $f \cap g$ is a 1-face.

Let $X \sqsubseteq \mathbb{F}_{2}^{n}$. A sequence $\pi=\left\langle f_{0}, \ldots, f_{l}\right\rangle$ of 2 -faces in $X$ is a strong path in $X$ (from $f_{0}$ to $f_{l}$ ) if $f_{i}$ and $f_{i+1}$ are strongly adjacent for each $i=0, \ldots, l-1$; the number $l$ is the length of $\pi$. We say that $X$ is strongly connected if, for any pair of 2 -faces $(f, g)$ in $X$, there is a strong path in $X$ from $f$ to $g$.

If $f$ is a 2-face of $\mathbb{F}_{2}^{n}$, we set:

$\Gamma^{*}(f)=\left\{g \in \mathbb{F}_{2}^{n} \mid g\right.$ is a 2 -face adjacent to $\left.f\right\}, \Gamma(f)=\Gamma^{*}(f) \cup\{f\}$; and $\Gamma_{\mathcal{S}}^{*}(f)=\left\{g \in \mathbb{F}_{2}^{n} \mid g\right.$ is strongly adjacent to $\left.f\right\}, \Gamma_{\mathcal{S}}(f)=\Gamma_{\mathcal{S}}^{*}(f) \cup\{f\}$. 


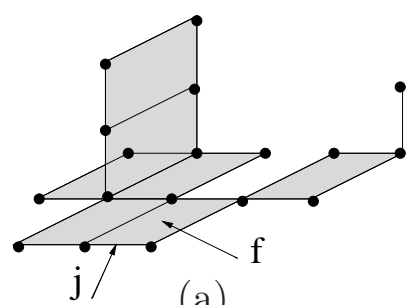

(a)

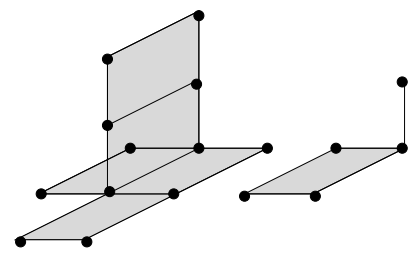

(d)

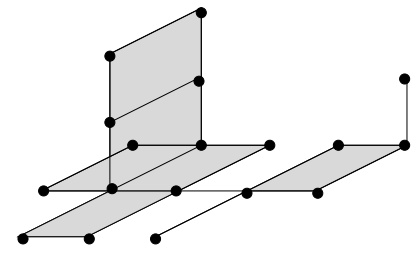

(b)

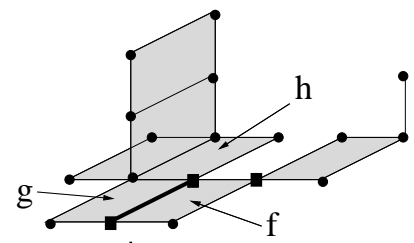

(e)

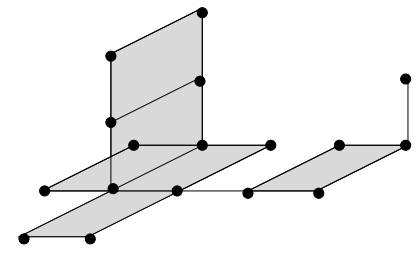

(c)

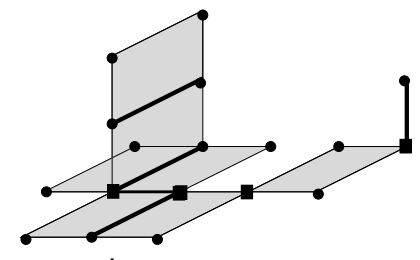

(f)

Fig. 3. (a) A complex $X$, (b) and (c) two steps of elementary collapse of $X$, (d) the detachment of $\hat{f}$ from $X$, (e) the attachment of the 2-face $f$ is highlighted, the face $f$ is not simple, whereas $g$ and $h$ are simple, (f) the essential 0 - and 1-faces for $X$ are highlighted.

\section{Simple cells}

Intuitively a cell $\hat{f}$ of a complex $X$ is simple if its removal from $X$ "does not change the topology of $X$ ". In this section we propose a definition of a simple cell based on the operation of collapse [13], which is a discrete analogue of a continuous deformation (a homotopy). Note that this definition is a rather general one, in particular, it may be directly extended to $n$-dimensional cubical complexes [5].

Let $X$ be a complex in $\mathbb{F}_{2}^{n}$ and let $f \in X^{+}$. The face $f$ is a border face for $X$ if there exists one face $g \in \hat{f}^{*}$ such that $f$ is the only face of $X$ which contains $g$. Such a face $g$ is said to be free for $X$ and the pair $(f, g)$ is said to be a free pair for $X$. We say that $f \in X^{+}$is an interior face for $X$ if $f$ is not a border face. In Fig. 3 (a), the pair $(f, j)$ is a free pair for $X$, and the complex $X$ has no interior face.

Let $X$ be a complex, and let $(f, g)$ be a free pair for $X$. The complex $X \backslash\{f, g\}$ is an elementary collapse of $X$.

Let $X, Y$ be two complexes. We say that $X$ collapses onto $Y$ if there exists a collapse sequence from $X$ to $Y$, i.e., a sequence of complexes $\left\langle X_{0}, \ldots, X_{l}\right\rangle$ such that $X_{0}=X, X_{l}=Y$, and $X_{i}$ is an elementary collapse of $X_{i-1}, i=1, \ldots, l$; the number $l$ is the length of the collapse sequence.

We say that a complex $X$ is collapsible if $X$ collapses onto a complex which is made of a single point.

In Fig. 3, a complex $X$ is depicted in (a), followed in (b) and (c) by two steps of elementary collapse. 
We give now a definition of a simple point, it may be seen as a discrete analogue of the one given by T.Y. Kong in [22] which lies on continuous deformations in the $n$-dimensional Euclidean space.

Definition 1. Let $X \preceq \mathbb{F}_{2}^{n}$. Let $f \in X^{+}$.

We say that $\hat{f}$ and $f$ are simple for $X$ if $X$ collapses onto $X \ominus \hat{f}$.

The notion of attachment, as introduced by T.Y. Kong [21,22], leads to a local characterization of simple cells.

Definition 2. Let $X \preceq \mathbb{F}_{2}^{n}$ and let $f \in X^{+}$.

The attachment of $\hat{f}$ for $X$ is the complex $\operatorname{Attach}(\hat{f}, X)=\hat{f}^{*} \cap[X \otimes \hat{f}]$.

In other words, a face $g$ is in $\operatorname{Attach}(\hat{f}, X)$ if $g$ is in $\hat{f}^{*}$ and if $g$ is a (proper) face of a facet $h$ distinct from $f$.

The following proposition is an easy consequence of the above definitions.

Proposition 3. Let $X \preceq \mathbb{F}_{2}^{n}$, and let $f \in X^{+}$.

The cell $\hat{f}$ is simple for $\bar{X}$ if and only if $\hat{f}$ collapses onto Attach $(\hat{f}, X)$.

The attachment of a 2 -face $f$ of a complex $X$ is highlighted Fig. 3 (e) and $X \odot \hat{f}$ is depicted in (d). It may be seen that $f$ is not simple: there is no collapse sequence from $X$ (a) to $X \odot \hat{f}$ (d). Observe that the complex is no more connected after the detachment of $\hat{f}$. On the other hand the faces $g$ and $h$ are simple.

The next property may be directly derived from Prop. 3.

Proposition 4. Let $X \preceq \mathbb{F}_{2}^{n}$, and let $f \in X^{+}$.

1) If $\hat{f}$ is a 0 -cell, then $\hat{f}$ is not simple for $X$;

2) If $\hat{f}$ is a 1-cell, then $\hat{f}$ is simple for $X$ if and only if $\operatorname{Attach}(\hat{f}, X)$ is made of a single point;

3) If $\hat{f}$ is a 2-cell, then $\hat{f}$ is simple for $X$ if and only if:

i) $f$ is a border face; and

ii) $\operatorname{Attach}(\hat{f}, X)$ is non-empty and connected.

From Prop. 4, we easily derive a characterization of simple 2-faces which is an equivalent, in the framework of $2 \mathrm{D}$ complexes in $\mathbb{F}_{2}^{n}$, of the well-known characterization of simple pixels in the square grid given by A. Rosenfeld [34].

Proposition 5. Let $X \sqsubseteq \mathbb{F}_{2}^{n}$, and let $f$ be a 2-face for $X$. The face $f$ is simple for $X$ if and only if:

i) $f$ is a border face; and

ii) $\Gamma^{*}(f) \cap X$ is non-empty and connected. 


\section{Critical kernels}

Let $X$ be a complex in $\mathbb{F}_{2}^{n}$. We observe that, if we remove simultaneously simple cells from $X$, we may obtain a set $Y$ such that $X$ does not collapse onto $Y$. In other words, if we remove simple cells in parallel, we may "change the topology" of the original object $X$. For example, in Fig. 3 (e), $g$ and $h$ are simple for $X$, but the complexes $X$ and $X \odot[\hat{g} \cup \hat{h}]$ have not "the same topology" (here, the same number of connected components). Thus, it is not possible to use directly the notion of simple cell for thinning discrete objects in a symmetrical manner.

In this section, we introduce a new framework for thinning in parallel discrete objects with the warranty that we do not alter the topology of these objects. This method may be extended for complexes of arbitrary dimension [5]. As far as we know, this is the first method which allows to thin arbitrary complexes in a symmetric way.

This method is based solely on three notions, the notion of an essential face which allows to define the core of a face, and the notion of a critical face.

Definition 6. Let $X \preceq \mathbb{F}_{2}^{n}$ and let $f \in X$. We say that $f$ is an essential face for $X$ if $f$ is precisely the intersection of all facets of $X$ which contain $f$, i.e., if $f=\cap\left\{g \in X^{+} \mid f \subseteq g\right\}$. We denote by $\operatorname{Ess}(X)$ the set composed of all essential faces of $X$. If $f$ is an essential face for $X$, we say that $\hat{f}$ is an essential cell for $X$.

Observe that a facet of $X$ is necessarily an essential face for $X$, i.e., $X^{+} \subseteq$ $\operatorname{Ess}(X)$. Observe also that the non-empty intersection of any number of facets is an essential face. The essential 0 - and 1-faces of the complex $X$ of Fig. 3 (a) are highlighted Fig. 3 (f).

Definition 7. Let $X \preceq \mathbb{F}_{2}^{n}$ and let $f \in E s s(X)$. The core of $\hat{f}$ for $X$ is the complex, denoted by $\operatorname{Core}(\hat{f}, X)$, which is the union of all essential cells for $X$ which are in $\hat{f}^{*}$, i.e., $\operatorname{Core}(\hat{f}, X)=\cup\left\{\hat{g} \mid g \in \operatorname{Ess}(X) \cap \hat{f}^{*}\right\}$.

The preceding definition may be seen as a generalization of the notion of attachment for arbitrary essential cells (which are not necessarily facets).

Proposition 8. Let $X \preceq \mathbb{F}_{2}^{n}$ and let $f$ be a facet of $X$. The attachment of $\hat{f}$ for $X$ is precisely the core of $\hat{f}$ for $X$, i.e, we have $\operatorname{Attach}(\hat{f}, X)=\operatorname{Core}(\hat{f}, X)$.

Definition 9. Let $X \preceq \mathbb{F}_{2}^{n}$ and let $f \in X$. We say that $f$ and $\hat{f}$ are regular for $X$ if $f \in \operatorname{Ess}(X)$ and if $\hat{f}$ collapses onto $\operatorname{Core}(\hat{f}, X)$. We say that $f$ and $\hat{f}$ are critical for $X$ if $f \in E s s(X)$ and if $f$ is not regular for $X$. 
We set $\operatorname{Critic}(X)=\cup\{\hat{f} \mid f$ is critical for $X\}, \operatorname{Critic}(X)$ is a complex that we call the critical kernel of $X$. A face $f$ in $X$ is a maximal critical face, or an $M$-critical face for $X$, if $f$ is a facet of $C r i t i c(X)$.

Again, the preceding definition of a regular cell is a generalization of the notion of a simple cell. As a corollary of Prop. 8, we have:

Proposition 10. Let $X \preceq \mathbb{F}_{2}^{n}$ and let $f$ be a facet of $X$. The cell $\hat{f}$ is regular for $X$ if and only if $\hat{f}$ is simple for $X$.

Furthermore, a face $f$ is regular if and only if $f$ is simple after removing all cells which contain $f$ while keeping the cell $\hat{f}$ :

Proposition 11. Let $X \preceq \mathbb{F}_{2}^{n}$ and let $f \in E s s(X)$. The cell $\hat{f}$ is regular for $X$ if and only if $\hat{f}$ is simple for $X^{\prime}=[X \otimes Y] \cup \hat{f}$, with $Y=\cup\left\{\hat{g} \mid g \in X^{+}\right.$ and $f \subseteq g$ \}.

We propose the following classification of critical faces which is specific to the $2 \mathrm{D}$ case. This classification is made according to the "topological type" of a critical face.

Definition 12. Let $X \preceq \mathbb{F}_{2}^{n}$, and let $f \in \operatorname{Ess}(X)$.

i) $f$ is $T_{0}$-critical for $X$ if $\operatorname{Core}(\hat{f}, X)=\emptyset$;

ii) $f$ is $T_{1}$-critical for $X$ if $\operatorname{Core}(\hat{f}, X)$ is not connected;

iii) $f$ is $T_{2}$-critical for $X$ if $f$ is an interior 2 -face.

From Prop. 4 and 11, a face $f$ is critical for $X \preceq \mathbb{F}_{2}^{n}$ if and only if $f$ is $T_{k}$-critical for some $k \in\{0,1,2\}$.

Let $X \preceq \mathbb{F}_{2}^{n}$ and let $f$ be an M-critical face for $X$. It means that $f$ is critical for $X$ and $f$ is not a proper face of a face which is critical for $X$. Let us denote by $D$ the complex which is the closure of the set composed of all faces which contain $f$ (including $f$ ).

Informally, if we "delete $D$ from $X$ ", i.e., if we transform $X$ into $X \ominus D$, then: i) We delete a connected component (a $0 \mathrm{D}$ cycle) of $X$ if $f$ is a $T_{0}$-critical face; ii) We split (create) a connected component or we delete 1D cycles (holes if $n=2$, tunnels if $n=3$ ) if $f$ is a $T_{1}$-critical face;

iii) We create a $1 \mathrm{D}$ cycle or we delete $2 \mathrm{D}$ cycles (which induce cavities if $n=3$ ) if $f$ is a $T_{2}$-critical face.

The following theorem holds for complexes of arbitrary dimensions (see [5]), it may be proved quite in a simple manner in the $2 \mathrm{D}$ case (first, we collapse regular 2-faces onto their core, then we collapse regular 1-faces onto their core). This is our basic result in this framework. 


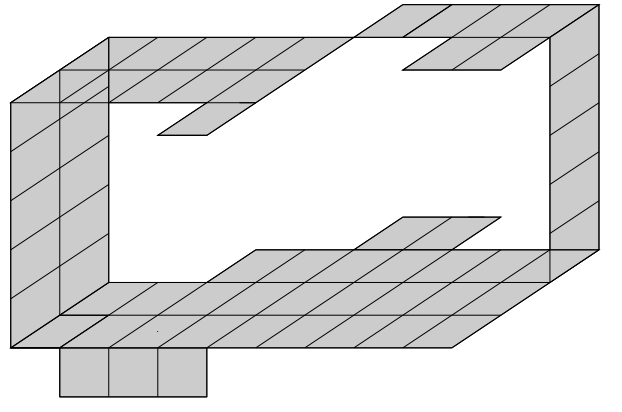

(a)

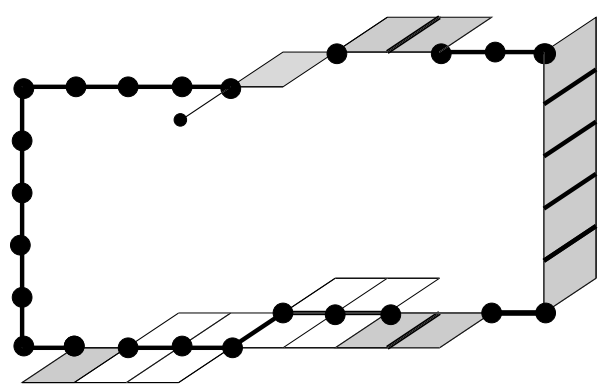

(c)

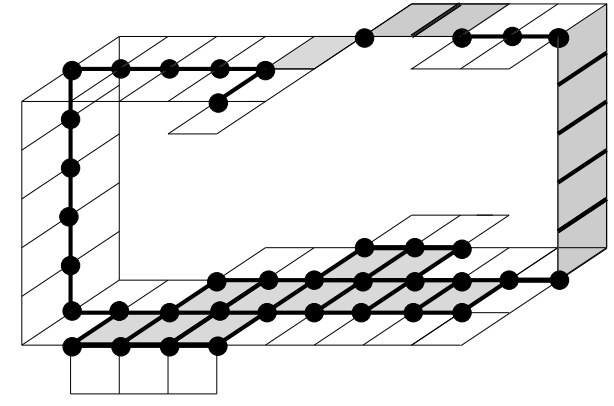

(b)

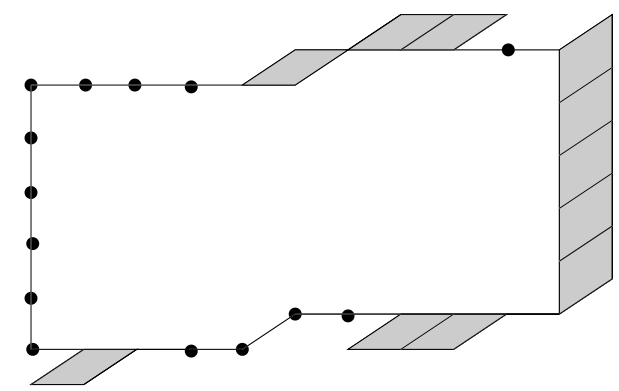

(d)

Fig. 4. (a): a complex $X_{0}$ in $\mathbb{F}_{2}^{3}$. (b): highlighted, $X_{1}=\operatorname{Critic}\left(X_{0}\right)$. (c): highlighted, $X_{2}=\operatorname{Critic}\left(X_{1}\right)$. (d): $X_{2}$ is such that $\operatorname{Critic}\left(X_{2}\right)=X_{2}$.

Theorem 13. Let $X \preceq \mathbb{F}_{2}^{n}$. The complex $X$ collapses onto its critical kernel. Furthermore, if $Y \sqsubseteq X$ is such that $Y$ contains the critical kernel of $X$, then $X$ collapses onto $Y$.

In Fig. 4 is depicted a complex $X_{0} \preceq \mathbb{F}_{2}^{3}$, as well as $X_{1}=\operatorname{Critic}\left(X_{0}\right)$ and $X_{2}=\operatorname{Critic}\left(X_{1}\right)$. The complex $X_{2}$ is such that $\operatorname{Critic}\left(X_{2}\right)=X_{2}$.

\section{Crucial kernels}

If $X$ is a complex in $\mathbb{F}_{2}^{n}$, the subcomplex $\operatorname{Critic}(X)$ is not necessarily a principal subcomplex of $X$, as illustrated Fig. 4. In this paper we investigate thinning algorithms which take as input a pure 2-complex and which return a principal subcomplex of the input (thus also a pure 2-complex). In this section, we propose some notions which allow to recover a principal subcomplex $Y$ of an arbitrary complex $X$, with the constraint that $X$ collapses onto $Y$.

Definition 14. Let $X \preceq \mathbb{F}_{2}^{n}$, and let $f \in X^{+}$be a simple facet for $X$.

We say that $f$ and $\hat{f}$ are crucial for $X$, if $\hat{f}^{*}$ contains a face which is M-critical for $X$. We say that $f$ and $\hat{f}$ are $T_{k}$-crucial for $X$, if $\hat{f}^{*}$ contains an M-critical face which is $T_{k}$-critical for $X, k=0,1$. 


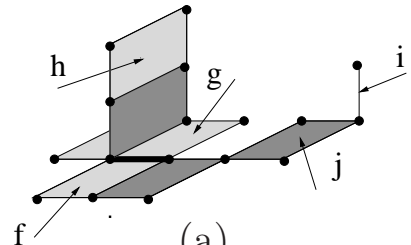

(a)

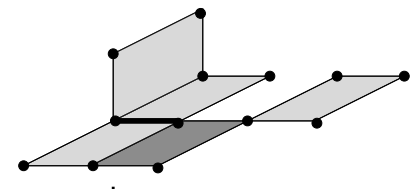

(b)

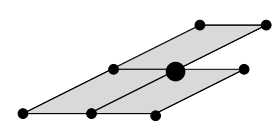

(c)

Fig. 5. (a): A complex $X_{0}$ and its M-critical faces (highlighted). (b): $X_{1}=\operatorname{Cruc}\left(X_{0}\right)$ and its M-critical faces. (c): The complex $X_{2}=\operatorname{Cruc}\left(X_{1}\right)$ contains only one M-critical face (highlighted), and $X_{2}=\operatorname{Cruc}\left(X_{2}\right)$.

Thus, a critical face for $X$ is either a facet which is not simple, or is included in a crucial face (which is a simple facet).

In Fig. 5 (a), the M-critical faces of a complex are highlighted. The faces $f$ and $g$ are crucial ( $T_{1}$-crucial), the faces $i$ and $h$ are simple but not crucial (the critical faces included in $i$ and $h$ are not M-critical), the face $j$ is not simple (it is M-critical), thus $j$ is not crucial.

Definition 15. Let $X \preceq \mathbb{F}_{2}^{n}$, and let $K$ be a set of crucial faces for $X$.

We say that $K$ is a $\left(T_{k^{-}}\right)$crucial clique for $X$, if there exists a $\left(T_{k^{-}}\right.$-critical) face $f$ which is M-critical for $X$ and such that $K$ is precisely the set of facets of $X$ which contain $f$. We also say that $K$ is the crucial clique induced by $f$.

In Fig. 5 (a), the set of faces $K=\{f, g\}$ is a $T_{1}$-crucial clique, in (c) the set $K^{\prime}$ composed of the three 2 -faces is a $T_{0}$-crucial clique.

Definition 16. Let $X \preceq \mathbb{F}_{2}^{n}$ and let $Y \sqsubseteq X$.

We say that $Y$ is a crucial retraction of $X$ if:

i) $Y$ contains each facet of $X$ which is critical; and

ii) $Y$ contains at least one face of each crucial clique for $X$.

From the above definitions, we immediately derive the following property.

Proposition 17. Let $X \preceq \mathbb{F}_{2}^{n}$ and let $Y \sqsubseteq X$.

We have Critic $(X) \subseteq Y$ if and only if $Y$ is a crucial retraction of $X$.

Thus, by Th. 13, if $Y$ is a crucial retraction of $X$, then $X$ collapses onto $Y$. All algorithms proposed in this paper will iteratively compute crucial retractions.

Let us define the crucial kernel of $X$ as the set $\operatorname{Cruc}(X)$ which is the union of all cells of $X$ which are either critical or crucial for $X$. In Fig. 5 (a), a complex $X_{0}$ and its M-critical faces (three 2-faces and one 1-face) are depicted. The complex $X_{1}=\operatorname{Cruc}\left(X_{0}\right)$ is given in (b) also with its M-critical faces (one 2 -face and one 1-face, which are both $T_{1}$-critical). Finally, in (c), the complex $X_{2}=\operatorname{Cruc}\left(X_{1}\right)$ contains only one M-critical face (which is $T_{0}$-critical), and it may be seen that $X_{2}=\operatorname{Cruc}\left(X_{2}\right)$. 
For thinning objects, we often want to keep other faces than the ones which are either not simple or crucial. That is why we introduce the following definition; intuitively, the set $K$ corresponds to a set we want to preserve during a thinning procedure (like extremities of curves, if we want to obtain a curvilinear skeleton).

Definition 18. Let $X \preceq \mathbb{F}_{2}^{n}$, and $K$ be a set of facets of $X$. Let $R_{0}$ be the set of all facets of $X$ which are critical for $X$, we set $R_{1}=K \cup R_{0}$. We say that a facet $f$ of $X$ is $\left(T_{k^{-}}\right)$crucial for $\langle X, K\rangle$, if $f$ belongs to a $\left(T_{k^{-}}\right)$crucial clique for $X$ which is included in $X^{+} \backslash R_{1},(k=0,1)$. Let $R_{2}$ be the set of all faces which are crucial for $\langle X, K\rangle$. The complex $R_{1}^{-} \cup R_{2}^{-}$is the crucial kernel of $X$ constrained by $K$.

Observe that a face is crucial for $X$ if and only if it is crucial for $\langle X, K\rangle$, where $K$ is the empty set.

Remark: The preceding definition will be a "template" for all the thinning algorithms presented hereafter: see the expression of these algorithms proposed in the next sections. In fact, all our algorithms iteratively compute, until idempotence, such constrained crucial kernels.

From the previous definitions, we immediately deduce the following proposition which ensures that any constrained crucial kernel preserves topology.

Proposition 19. Let $X \preceq \mathbb{F}_{2}^{n}$, and let $K$ be a set of facets of $X$. The crucial kernel of $X$ constrained by $K$ is a crucial retraction of $X$.

\section{Combinatorial characterizations of crucial faces in $\mathbb{F}_{2}^{2}$}

In order to design efficient parallel thinning algorithms, we need some characterizations of crucial faces which may be easily checked by inspecting a limited neighborhood of each face. For that purpose, we will examine the possible configurations of the 2 -faces which contain an M-critical $k$-face, $k=0,1$. We focus on the important particular case of $\mathbb{F}_{2}^{2}$ (a discrete plane).

Lemma 20. Let $X \sqsubseteq \mathbb{F}_{2}^{2}$, and let $f$ be a 1-face of $X$. Without loss of generality (up to a $\pi / 2$ rotation) let us assume that the neighborhood of $f$ is depicted by Fig. 6(a). The face $f$ is $M$-critical for $X$ if and only if:

i) $a_{1} \in X$ and $a_{2} \in X$; and

ii) $a_{1}$ and $a_{2}$ are both simple for $X$; and

iii) either $\left\{b_{1}, b_{2}, b_{3}, b_{4}\right\} \cap X=\emptyset$ or $\left(\left\{b_{1}, b_{2}\right\} \cap X \neq \emptyset\right.$ and $\left.\left\{b_{3}, b_{4}\right\} \cap X \neq \emptyset\right)$.

Proof: Suppose that $f$ is M-critical for $X$. Since $f$ has to be essential, both $a_{1}$ 
and $a_{2}$ are in $X$, hence i). By the very definition of an M-critical face, we must also have ii). Since $f$ is critical for $X, c_{1}$ and $c_{2}$ are either both essential or both non-essential for $X$, hence condition iii). The proof that the conjunction of i), ii) and iii) is a sufficient condition is straightforward.

For the three following lemmas, we use the naming conventions depicted in Fig. 6(b). We prove only the necessary conditions, the sufficient conditions are straightforward.

Lemma 21. Let $X \sqsubseteq \mathbb{F}_{2}^{2}$, and let $f$ be a 0-face of $X$ which is included in exactly two 2-faces of $X$. The face $f$ is $M$-critical for $X$ if and only if:

i) The two 2-faces of $X$ that contain $f$ are either $\left\{a_{0}, a_{2}\right\}$ or $\left\{a_{1}, a_{3}\right\}$; and

ii) The two 2-faces of $X$ that contain $f$ are simple for $X$.

Proof: The two 2-faces which contain $f$ cannot be $\left\{a_{0}, a_{1}\right\}$ (or similar cases up to $\pi / 2$ rotations), otherwise the face $f$ would not be essential, hence not critical for $X$. Furthermore, whatever these 2-faces, they cannot be critical for $X$ (by definition of an M-critical face).

Lemma 22. Let $X \sqsubseteq \mathbb{F}_{2}^{2}$, and let $f$ be a 0 -face of $X$ which is included in exactly three 2-faces of $X$. Without loss of generality (up to $\pi / 2$ rotations), assume that these three 2-faces are $a_{0}, a_{1}$ and $a_{2}$. The face $f$ is $M$-critical for $X$ if and only if:

i) $a_{i}$ is simple for $X$, for any $i=0,1,2$; and

ii) $d_{i} \notin X$, for any $i=1,2,3,4$.

Proof: If $f$ is M-critical, we must have condition $\mathrm{i}$ ), and also the faces $b_{i}$ must be not critical for $X$. It can be seen that $b_{1}, b_{2}$ are essential, thus they must also be regular for $X$. It implies that the faces $c_{1}, c_{2}$ must be non-essential, hence condition ii).

Lemma 23. Let $X \sqsubseteq \mathbb{F}_{2}^{2}$, and let $f$ be a 0 -face of $X$ which is included in four 2-faces of $X$. The face $f$ is $M$-critical for $X$ if and only if:

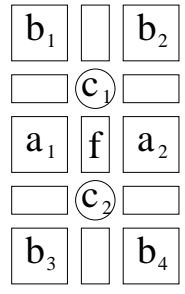

(a)

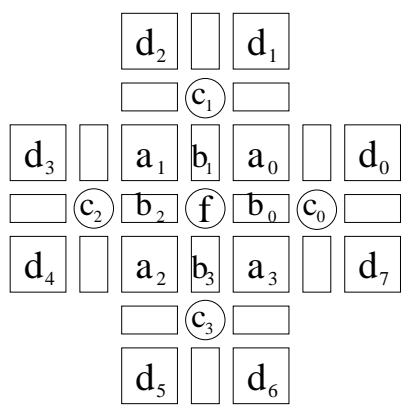

(b)

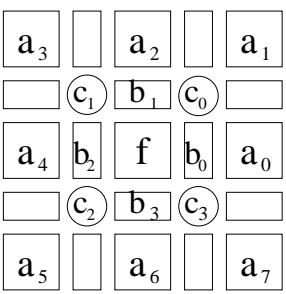

(c)

Fig. 6. Naming conventions for Lemmas 20-23 and Lemmas 48-49. 


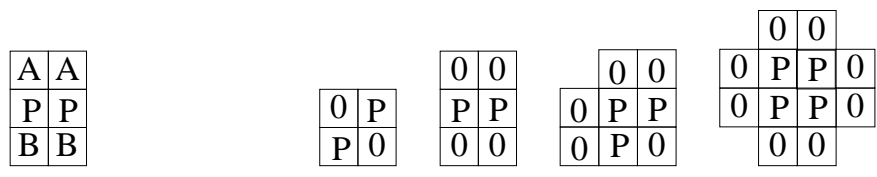

$$
\begin{aligned}
& \begin{array}{llllll}
C & C_{1} & C_{2} & C_{3} & C_{4}
\end{array}
\end{aligned}
$$

Fig. 7. Patterns and masks for crucial pixels. The 11 masks corresponding to these 5 patterns are obtained from them by applying any series of $\pi / 2$ rotations. The label 0 indicates pixels that must belong to the complement of $S$. The label $P$ indicates pixels that must belong to the set $P$ which is a set composed of simple pixels of $S$. For mask $C$, at least one of the pixels marked A and at least one of the pixels marked B must be in $S$. If one of these masks matches the sets $S, P$, then all the pixels which correspond to a label $P$ in the mask are recorded as "matched".

i) $a_{i}$ is simple for $X$, for any $i=0,1,2,3$; and

ii) $d_{i} \notin X$, for any $i=0, \ldots, 7$.

Proof: If $f$ is M-critical for $X$, we must have condition i), and also the faces $b_{i}$ must be not critical for $X$. It can be seen that the $b_{i}$ are all essential, thus they must be regular for $X$. It means that the faces $c_{i}$ must all be non-essential, hence condition ii).

\section{Crucial pixels in the square grid}

We introduce the following definitions in order to establish a link between planar pure complexes (i.e., pure 2-complexes in $\mathbb{F}_{2}^{2}$ ) and the square grid as considered in image processing $[34,36,25]$.

We define the square grid as the set $\mathbb{G}^{2}$ composed of all 2-faces of $\mathbb{F}_{2}^{2}$. A 2-face of $\mathbb{G}^{2}$ is also called a pixel. In the sequel, we will consider only finite subsets of $\mathbb{G}^{2}$.

For any pure 2-complex in $\mathbb{F}_{2}^{2}$, i.e., for any $X \sqsubseteq \mathbb{F}_{2}^{2}$, we associate the subset $X^{+}$of $\mathbb{G}^{2}$. In return, to each finite subset $S$ of $\mathbb{G}^{2}$, we associate the complex $S^{-}$in $\mathbb{F}_{2}^{2}$. In the sequel, this will be our basic methodology to "interpret" a set of pixels. In particular, all definitions given for a facet in $X^{+}$have their counterparts for a pixel in $\mathbb{G}^{2}$. For example if $S \subseteq \mathbb{G}^{2}$ and $p \in S$, we will say that the pixel $p$ is simple for $S$ if $p$ is simple for $S^{-}$. Border, interior, $\left(T_{k^{-}}\right)$ critical, and $\left(T_{k^{-}}\right)$crucial pixels are defined in the same manner. If $K$ is a set made of pixels of $S$, we say that $p$ is crucial for $\langle S, K\rangle$ if $p$ is crucial for $\left\langle S^{-}, K\right\rangle$.

Observe that, if $p \in \mathbb{G}^{2}, \Gamma^{*}(p)$ and $\Gamma_{\mathcal{S}}^{*}(p)$ correspond to the so-called 8neighborhood and 4-neighborhood of $p$, respectively. 
Thanks to the combinatorial characterizations of Section 5, we can give some simple local conditions, in the square grid, for crucial pixels. We express these local conditions by a set of masks, as in most papers related to parallel thinning in the digital topology framework.

The following property is a direct consequence of lemmas 20, 21, 22 and 23. The definition of the masks $C, C_{1}, \ldots, C_{4}$ is given Fig. 7 .

Proposition 24. Let $S \subseteq \mathbb{G}^{2}$, and $p \in S$. Let $P$ be a set composed of simple pixels of $S$.

i) The pixel $p$ belongs to a $T_{1}$-crucial clique included in $P$ if and only if $p$ is matched by pattern $C$;

ii) The pixel $p$ belongs to a $T_{0}$-crucial clique included in $P$ if and only if $p$ is matched by one of the patterns $C_{1}, \ldots C_{4}$.

Thus, the mask $C$ is a mask for $T_{1}$-crucial cliques, and $C_{1}, \ldots, C_{4}$ are masks for $T_{0}$-crucial cliques. For each of these masks, the crucial clique is the set composed of $P$ 's. In fact these masks are also masks for the minimal non-simple sets introduced by $\mathrm{C}$. Ronse [33], see section 14 . We observe that, since $P$ is composed of simple pixels of $S$, the set of $P$ 's of each mask $C_{1}, \ldots, C_{4}$ is necessarily surrounded by 0 's. Hence, we have the following property.

Proposition 25. Let $S \subseteq \mathbb{G}^{2}$, and let $U$ be a $T_{0}$-crucial clique for $S$. Then $U$ is a connected component of $S$.

\section{$7 \quad$ Minimal $\mathcal{K}$-skeletons}

A minimal symmetric skeleton of an object may be obtained by deleting iteratively, in parallel, all pixels which are neither critical nor crucial.

Definition 26. Let $S \subseteq \mathbb{G}^{2}$. The crucial kernel of $S$ is the set $\operatorname{Cruc}(S)$ which is composed of all critical pixels and all crucial pixels of $S$.

Let $\left\langle S_{0}, S_{1}, \ldots, S_{k}\right\rangle$ be the unique sequence such that $S_{0}=S, \operatorname{Cruc}\left(S_{k}\right)=S_{k}$ and $S_{i}=\operatorname{Cruc}\left(S_{i-1}\right), i=1, \ldots, k$. The set $S_{k}$ is the minimal $\mathcal{K}$-skeleton of $S$.

By Prop. 19 (here $K=\emptyset$ ), the minimal $\mathcal{K}$-skeleton of a set $S$ is a crucial retraction of $S$. The following algorithm computes a minimal $\mathcal{K}$-skeleton.

Algorithm $M K_{a}^{2}$ (Input /Output : a set $S \subseteq \mathbb{G}^{2}$ )

01. Repeat Until Stability

02. $R_{1} \leftarrow$ set of pixels which are critical for $S$

03. $R_{2} \leftarrow$ set of pixels belonging to a $T_{0^{-}}$or $T_{1}$-crucial clique included in $S \backslash R_{1}$

04. $S \leftarrow R_{1} \cup R_{2}$ 


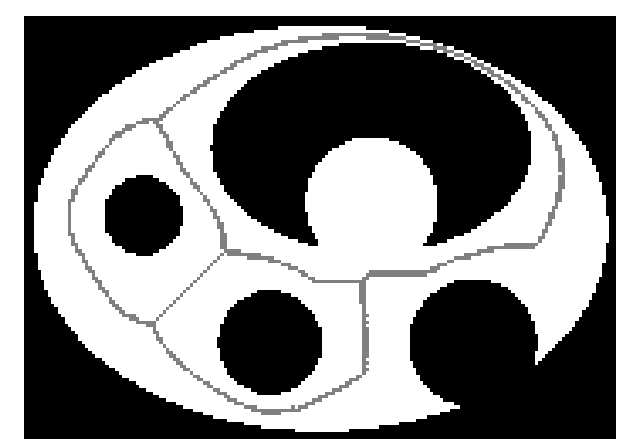

Fig. 8. A subset $S$ of $\mathbb{G}^{2}$ (in white) and its minimal $\mathcal{K}$-skeleton (in gray).

From Prop. 24, we may check if a pixel is $T_{1}$-crucial by using the pattern $C$. Considering all possible rotations, there are in fact only two masks corresponding to $C$. On the other hand, it may be seen that the checking of a $T_{0}$-crucial pixel with the patterns $C_{1}, \ldots, C_{4}$ involves 9 masks. In the following, we propose an algorithm which avoids the use of these 9 masks. This algorithm is based on a technic used for computing the so-called ultimate erosions in the context of mathematical morphology (see [40]).

Let $S \subseteq \mathbb{G}^{2}$, we denote by $S \ominus \Gamma^{*}=\left\{p \in S \mid \Gamma^{*}(p) \subseteq S\right\}$, the erosion of $S$ by $\Gamma^{*}$, and by $S \oplus \Gamma^{*}=\cup\left\{\Gamma^{*}(p) \mid p \in S\right\}$, the dilation of $S$ by $\Gamma^{*}$.

Algorithm $M K^{2}$ (Input /Output : a set $S \subseteq \mathbb{G}^{2}$ )

01. Repeat Until Stability

02. $R_{1} \leftarrow$ set of pixels which are critical for $S$

03. $\quad R_{2} \leftarrow$ set of pixels which belong to a $T_{1}$-crucial clique included in $S \backslash R_{1}$

04. $\quad T \leftarrow R_{1} \cup R_{2}$

05. $S \leftarrow T \cup\left[S \backslash\left(T \oplus \Gamma^{*}\right)\right]$

The correctness of the algorithm lies on the following property.

Proposition 27. Let $S \subseteq \mathbb{G}^{2}$, and let $p \in S$ be a simple pixel.

i) If $p$ is not crucial for $S$, then there exists $q \in \Gamma^{*}(p) \cap S$ such that $q$ is either critical or $T_{1}$-crucial for $S$.

ii) If $p$ is $T_{0}$-crucial for $S$, then any $q \in \Gamma^{*}(p) \cap S$ is neither critical, nor $T_{1}$-crucial.

Proof:

i) Let $p \in S$ be a simple pixel not crucial for $S$. Since $p$ is simple, we have $\Gamma^{*}(p) \cap S \neq \emptyset$. Let us consider the two sets $U=S \backslash \Gamma^{*}(p)$ and $V=S \backslash \Gamma(p)$. If any $q \in \Gamma^{*}(p) \cap S$ is neither critical nor crucial for $S$, by Th. 13, $S^{-}$ would collapse onto $U^{-}$and also onto $V^{-}$. But $U^{-}$has one more connected component than $V^{-}$, a contradiction with the fact that the collapse operation preserves the number of connected components. It follows that there exists $q \in \Gamma^{*}(p) \cap S$ such that $q$ is either critical or crucial for $S$. Now, $q$ cannot be $T_{0}$-crucial, otherwise, from Prop. $25, p$ would also be $T_{0}$-crucial. 
ii) is a direct consequence of Prop. 25 .

Let us denote by $M K^{2}(S)$ the result obtained by algorithm $M K^{2}$ from the input $S$. From Prop. 27, the pixels which are added to the set $T$ at step 05 of $M K^{2}$ are precisely $T_{0}$-crucial pixels. Thus, we have the following property.

Proposition 28. Let $S \subseteq \mathbb{G}^{2}$. The set $M K^{2}(S)$ is the minimal $\mathcal{K}$-skeleton of $S$.

An example of a minimal $\mathcal{K}$-skeleton is given Fig. 8. As far as we know, $M K^{2}$ is the first algorithm for a minimal symmetric skeleton. Furthermore, the result of $M K^{2}$ is an object which is well-defined. To our best knowledge, this is also the first attempt to give a precise definition of such a notion.

\section{Curvilinear $\mathcal{K}$-skeletons}

Curvilinear skeletons keep track of some geometrical information relative to elongated or salient parts of the shape. In many thinning algorithms, such a skeleton is obtained thanks to the preservation of "end pixels" which are usually defined through a set of masks. We propose a general (i.e., rather non combinatorial) definition of an end, which arises naturally in the framework of critical kernels.

Definition 29. Let $X \preceq \mathbb{F}_{2}^{n}$, and let $f \in X^{+}$. We say that the facet $f$ is an end face for $X$ if $\hat{f}^{*}$ contains exactly one critical face for $X$.

Let $S \subseteq \mathbb{G}^{2}$, let $p \in S$. We say that $p$ is an end for $S$ if $p$ is an end face for $S^{-}$.

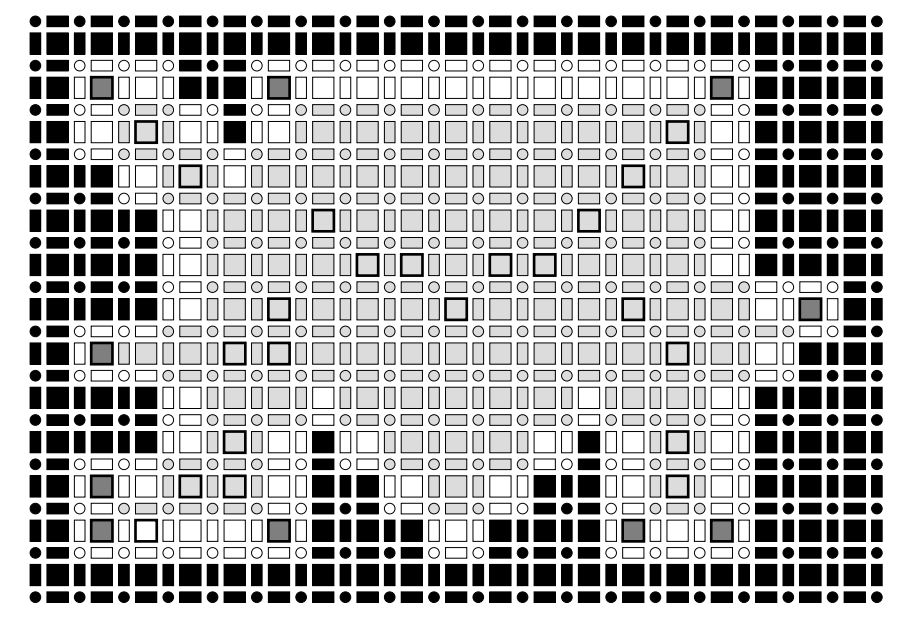

Fig. 9. A complex $X$ (all elements but the ones in black). In light gray: the critical faces for $X$. In dark gray: the end faces for $X$. The 2 -faces which correspond to medial axis elements (see section 10) are highlighted by a bold contour. 


$$
\begin{array}{|c|c|c|}
\hline 0 & \mathrm{~A} & \mathrm{~A} \\
\hline 0 & \mathrm{p} & \mathrm{A} \\
\hline 0 & 0 & 0 \\
\hline
\end{array}
$$

$E$

Fig. 10. Characterization of ends. The masks obtained from this one by $\pi / 2$ rotations must be added. The label 0 indicates pixels that must belong to the complement of $S$. At least one pixel labeled A must belong to $S$.

An illustration is given Fig. 9, where the critical faces are depicted in light gray and the end faces are in dark gray.

We follow the methodology of the preceding sections, by first establishing a combinatorial characterization of end faces in $\mathbb{F}_{2}^{2}$. This characterization is given in appendix A by the Lemmas 48 and 49. As for the crucial faces in Sec. 7, we can then characterize with a set of masks the pixels of an object in $\mathbb{G}^{2}$ which are ends. The following property is a direct consequence of these two lemmas, the definition of the mask $E$ is given Fig. 10 .

Proposition 30. Let $S \subseteq \mathbb{G}^{2}$, and let $p \in S$. The pixel $p$ is an end for $S$ if and only if the neighborhood of $p$ matches the mask $E$.

The next property allows to avoid the checking for $T_{0}$-crucial faces. It may be verified from Prop. 5 and by a direct inspection of the masks $E, C_{1}, \ldots, C_{4}$.

Proposition 31. Let $S \subseteq \mathbb{G}^{2}$, let $p \in S$.

i) If $p$ is an end for $S$, then $p$ is simple for $S$;

ii) If $p$ is $T_{0}$-crucial, then $p$ is an end.

We are now ready to formulate an algorithm. Let us denote by $E K^{2}(S)$ the result of the algorithm, $E K^{2}(S)$ is the $\mathcal{K}$-skeleton of $S$ based on ends. By Prop. 31 and $19, E K^{2}(S)$ is a crucial retraction of $S$. See Fig. 11 (a) for an example of such a skeleton.

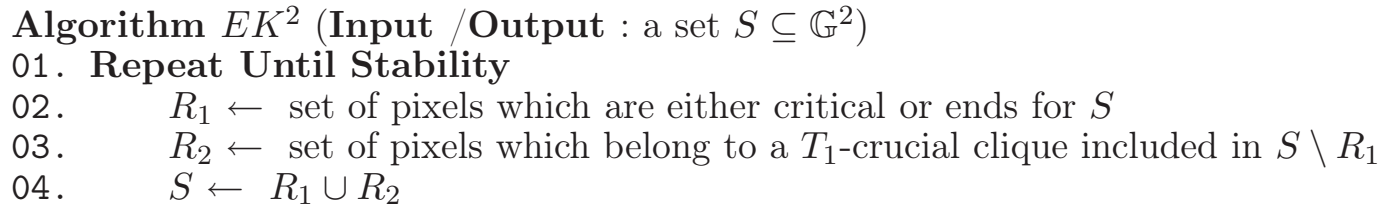

We will consider now another notion of extremity which is often used for thinning objects.

Definition 32. Let $S \subseteq \mathbb{G}^{2}$, let $p$ be a border pixel. We say that $p$ is residual $\left(\right.$ for $S$ ) if there is no interior pixel in $\Gamma_{\mathcal{S}}^{*}(p)$. 


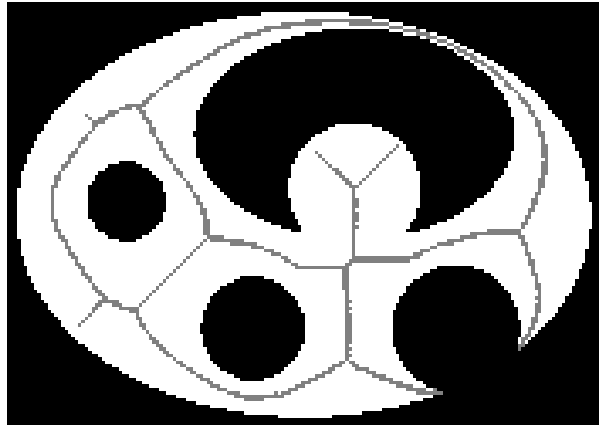

(a)

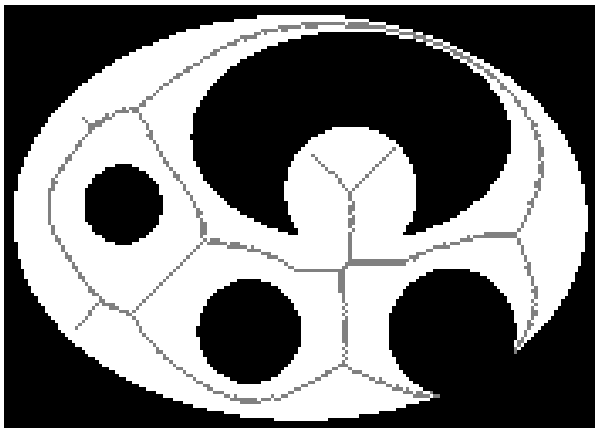

(b)

Fig. 11. A subset $S$ of $\mathbb{G}^{2}$ (in white), (a) its $\mathcal{K}$-skeleton based on ends, (b) its $\mathcal{K}$-skeleton based on residues.

We observe that a $T_{0}$-crucial pixel is necessarily a residual pixel (but here a residual pixel is not necessarily simple). Thus, we may give the same algorithm as above for computing $R K^{2}(S)$ which is the $\mathcal{K}$-skeleton of $S$ based on residues. See Fig. 11 (b) for an example.

Algorithm $R K^{2}$ (Input /Output : a set $S \subseteq \mathbb{G}^{2}$ )

01. Repeat Until Stability

02. $\quad R_{1} \leftarrow$ set of pixels which are either critical or residual for $S$

03. $\quad R_{2} \leftarrow$ set of pixels which belong to a $T_{1}$-crucial clique included in $S \backslash R_{1}$ 04. $S \leftarrow R_{1} \cup R_{2}$

Using the methodology of critical kernels, we may easily design parallel (here symmetrical) thinning algorithms based on different definitions of extremities. These algorithms clearly separate the topological and the geometrical conditions. It should be noted that this is not often the case, many parallel thinning algorithms do not make explicit these two kinds of conditions which are different in nature (see [10]).

\section{Topological axis and medial axis}

The quality of a curvilinear skeleton is often assessed by the fact that it contains, approximately or completely, the medial axis of the shape. We introduce the following definitions in order to show that there is a deep link between the medial axis and the collapse operation, and in order to generalize the medial axis for pure 2-complexes in $\mathbb{F}_{2}^{n}$, for arbitrary $n$.

Definition 33. Let $X \sqsubseteq \mathbb{F}_{2}^{n}$, and let $f \in X^{+}$. We set $\rho(f, X)$ as the minimum length of a collapse sequence of $X$ necessary to remove $f$ from $X$, if such a sequence exists, and $\rho(f, X)=\infty$ otherwise. We define the topo- 
logical axis of $X$ as the set of faces $f$ in $X^{+}$such that $\rho(f, X)=\infty$ or $\rho(f, X) \geq \max \left\{\rho(g, X) \mid g \in \Gamma_{\mathcal{S}}^{*}(f)\right.$ and $\left.\rho(g, X) \neq \infty\right\}$.

Note that we have $\rho(f, X)=1$ if and only if $f$ is a border face for $X$.

Let $X \sqsubseteq \mathbb{F}_{2}^{n}$, and let $f \in X^{+}$. We denote by $\pi^{\prime}(f, X)$ the length of a shortest strong path, in $X$, from $f$ to a border face of $X$, if such a path exists, and $\pi^{\prime}(f, X)=\infty$ otherwise. We denote by $\pi(f, X)$ the length of a shortest strong path, in $\mathbb{F}_{2}^{n}$, from $f$ to a border face of $X$.

We observe that $\rho(f, X)=\pi^{\prime}(f, X)+1$.

Now we focus our attention on the case $n=2$. Let $X \sqsubseteq \mathbb{F}_{2}^{2}$, and let $f \in X^{+}$. We have necessarily $\rho(f, X) \neq \infty$. Furthermore, since any 1 -face in $\mathbb{F}_{2}^{2}$ is included in precisely two 2-faces, it may be seen that $\pi(f, X)=\pi^{\prime}(f, X)$, thus $\rho(f, X)=\pi(f, X)+1$.

Let us recall a definition of the medial axis in the discrete grid $\mathbb{G}^{2}$ (see also [37]). Let $p, q \in \mathbb{G}^{2}$, we set $d(p, q)$ as the length of a shortest strong path from $p$ to $q$. This defines a distance (often called the 4-distance or the city block distance). Let $p \in \mathbb{G}^{2}$ and $r \geq 0$, we denote by $B_{r}(p)$ the ball of radius $r$ centered on $p$, defined by $B_{r}(p)=\left\{q \in \mathbb{G}^{2}, d(p, q) \leq r\right\}$. Observe that $B_{1}(p)=\Gamma_{\mathcal{S}}(p)$. Let $S \subseteq \mathbb{G}^{2}, p \in S, r \geq 0$. The ball $B_{r}(p) \subseteq S$ is maximal for $S$ if it is not strictly included in another ball included in $S$. The medial axis of $S$, denoted by $\operatorname{MA}(S)$, is the set of the centers of all the maximal balls for $S$. In [38], A. Rosenfeld and J.L. Pfaltz have proved that, for the city block and the chessboard distance, the medial axis of a shape can be obtained by detecting the local maxima of its distance transform. From the definition of the topological axis, and from the preceding remarks, we may deduce the following property which proves that the notion of topological axis indeed generalizes the one of medial axis (which is not defined for the case of two-dimensional structures in discrete $n$-dimensional spaces, $n>2$ ).

Proposition 34. Let $S \subseteq \mathbb{G}^{2}$. The medial axis of $S$ is precisely the topological axis of $S^{-}$.

In the framework of mathematical morphology, C. Lantuejoul gave the following characterization of the medial axis. Let $S \subseteq \mathbb{G}^{2}$, let $i \in \mathbb{N}$, we set $S \ominus B_{i}=\left\{p \in S \mid B_{i}(p) \subseteq S\right\}$, and $S \oplus B_{i}=\cup\left\{B_{i}(p) \mid p \in S\right\}$. Observe that $S \ominus B_{0}=S \oplus B_{0}=S$. We have (see [40]):

$$
\operatorname{MA}(S)=\cup\left\{\left[S \ominus B_{i}\right] \backslash\left[\left(S \ominus B_{i+1}\right) \oplus B_{1}\right] \mid i \in \mathbb{N}\right\}
$$

Let us examine now the $\mathcal{K}$-skeleton based on ends with respect to the medial axis. For that purpose, let us consider again Fig. 9. The pixels (2-faces) which belong to the medial axis are highlighted by a bold contour. We observe that all end faces (in dark gray) belong to the medial axis. We also observe that 


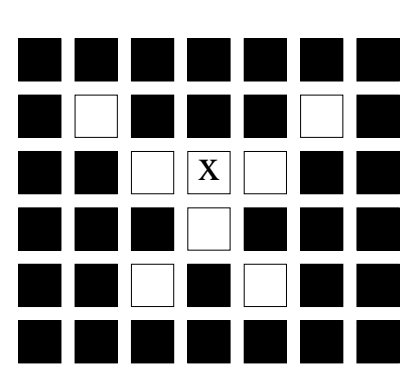

(a)

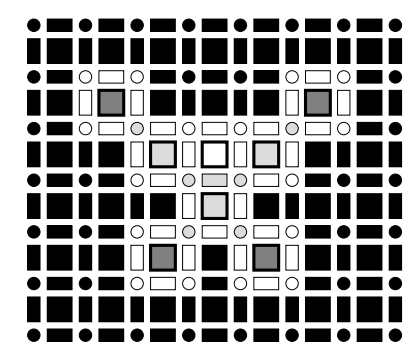

(b)

Fig. 12. (a): A subset $S$ of $\mathbb{G}^{2}$ (in white) which is equal to its medial axis $\operatorname{MA}(S)$. (b): The complex $X=S^{-}$(all elements but the ones in black). In light gray: the critical faces for $X$. In dark gray: the end faces for $X$.

there is a simple 2-face (in white), at the bottom left of the shape, which corresponds to a medial axis element but which is not an end face. In this case, the pixel will be preserved by algorithm $E K^{2}$ because it is a crucial pixel for $\langle S, K\rangle$ where $K$ is the set of end pixels. But in fact this is not always the case. Despite the appearances, the $\mathcal{K}$-skeleton based on ends of the object depicted Fig. 11 (a) does not contain all pixels of its medial axis, this axis is depicted Fig. 13 (a). There are three pixels of the medial axis which are not in this skeleton. The simpler example of Fig. 12 shows a case where a pixel $x$ belongs to the medial axis but is neither an end, nor a crucial pixel for $\langle S, K\rangle$.

In Appendix C (Fig. 21), we may find the number of medial axis pixels which are contained in three $\mathcal{K}$-skeletons based on ends ( $E K^{2}$ algorithm) and on residual pixels ( $R K^{2}$ algorithm). By the very definition of a residual pixel, at the first iteration of $R K^{2}$, the residual pixels are precisely the pixels of the medial axis which are border pixels. Thus, we could think that $R K^{2}$ better preserves the medial axis. In fact the results given in Fig. 21 indicate that this is not always the case. An example where $R K^{2}$ removes a medial axis pixel is given in Appendix B (Fig. 20).

\section{$10 \mathcal{K}$-skeletons and medial axis}

For obtaining a skeleton which includes the medial axis of the original object, we define the following notion of $\mathcal{K}$-skeleton which is constrained to include a given set $K$. According to Def. 18, if $S \subseteq \mathbb{G}^{2}$ and $K \subseteq S$, the crucial kernel of $S$ constrained by $K$ is the set which is the union of the set $K$, the set $R_{0}$ composed of all critical pixels, and the set of all pixels which belong to a crucial clique included in $S \backslash\left(K \cup R_{0}\right)$.

Definition 35. Let $S \subseteq \mathbb{G}^{2}$ and let $K \subseteq S$. We denote by $\operatorname{Cruc}(S, K)$ the crucial kernel of $S$ constrained by $K$. Let $\left\langle S_{0}, S_{1}, \ldots, S_{k}\right\rangle$ be the unique sequence 
such that $S_{0}=S, S_{k}=\operatorname{Cruc}\left(S_{k}, K\right)$ and $S_{i}=\operatorname{Cruc}\left(S_{i-1}, K\right), i=1, \ldots, k$. The set $S_{k}$ is the $\mathcal{K}$-skeleton of $S$ constrained by $K$.

By Prop 19, the $\mathcal{K}$-skeleton of a set $S$ constrained by a set $K$ is a crucial retraction of $S$. We give now a general result on constrained thinning which permits, under some conditions, to avoid the checking of the 9 masks (corresponding to $\left.C_{1}, \ldots, C_{4}\right)$ for the detection of $T_{0}$-crucial pixels. This result is a direct consequence of Prop. 25.

Proposition 36. Let $S \subseteq \mathbb{G}^{2}$. Let $K \subseteq S$, such that each connected component of $S$ contains at least one pixel of $K$. Then, it is not possible that there is a $T_{0}$-crucial clique for $S$ which is included in $S \backslash K$.

For computing a $\mathcal{K}$-skeleton constrained by the medial axis, we could first extract the medial axis, and then compute the constrained skeleton, this method is followed by B.K. Jang and R.T. Chin [19]. We present here an algorithm which computes at the same time the medial axis and the skeleton.

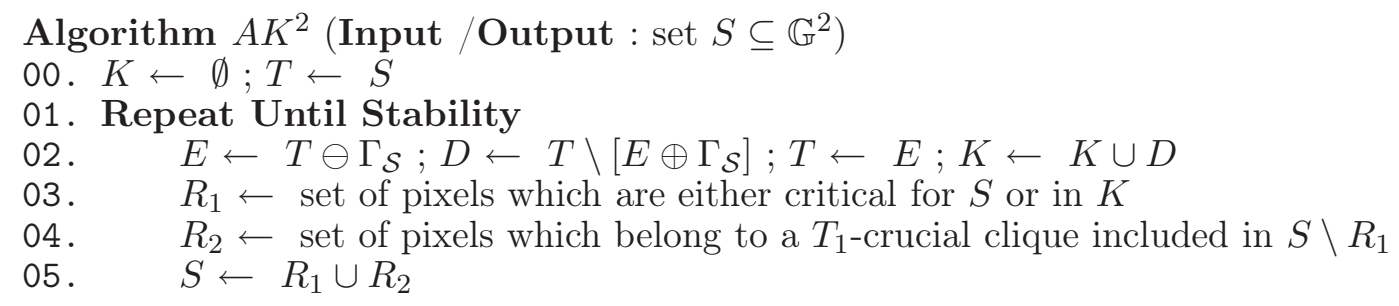

If we denote by $A K^{2}(S)$ the result obtained by algorithm $A K^{2}$, we then have the following property.

Proposition 37. Let $S \subseteq \mathbb{G}^{2}$. The set $A K^{2}(S)$ is the $\mathcal{K}$-skeleton of $S$ constrained by the medial axis of $S$.

Proof: Let us denote by $A M$ the medial axis of the original set $S$. It can be easily seen, from Eqn. (1), that the pixels which are accumulated in the set $K$ all belong to $A M$. Furthermore, at any step of the execution, any pixel of $A M$ which is simple for the current set $S$ is in the set $K$. Thus, at each step 04 of $A K^{2}$, the set $S \backslash R_{1}$ is composed precisely of the pixels of $S \backslash A M$ which are simple for $S$. Now, by the very definition of the medial axis, we are in the conditions of Prop. 36. Hence, at each step 05 of $A K^{2}$, all crucial pixels are preserved. This implies that the result of $A K^{2}$ is precisely the $\mathcal{K}$-skeleton of the original set $S$ constrained by its medial axis.

In Fig. 13, we show a subset $S$ of $\mathbb{G}^{2}$ together with its medial axis (a) and its medial $\mathcal{K}$-skeleton $A K^{2}(S)$ (b). In Fig. 21, we see that the result produced by $A K^{2}$ contains more pixels than the one of Jang and Chin's algorithm [19]. This is explained by the fact that this latter algorithm is not completely symmetri- 


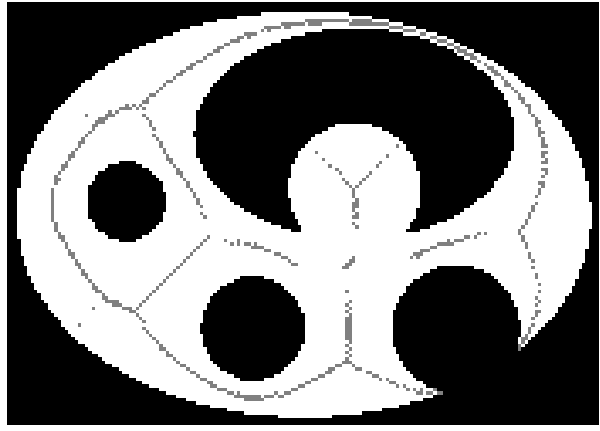

(a)

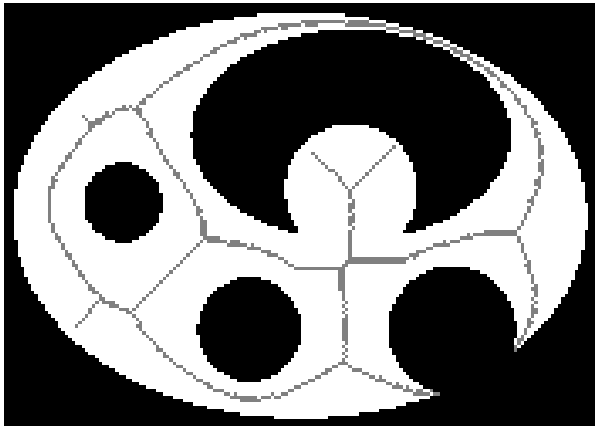

(b)

Fig. 13. (a): a subset $S$ of $\mathbb{G}^{2}$ (in white) and its medial axis $\mathrm{MA}(S)$ (in gray). (b): in gray, $A K^{2}(S)$.

cal (see algorithm $N K^{2}$ below). The algorithm proposed by T. Pavlidis [31,32], in its "reconstructing" variant, was designed to ensure the reconstruction of the original object (and the preservation of the medial axis). In fact this is not always the case, a commented counter-example may be found in [10] (see also Fig. 21). As far as we know, $A K^{2}$ is the first algorithm for a symmetric skeleton which contains the medial axis.

At each step of algorithm $A K^{2}$, a pixel which is not strongly adjacent to a pixel in $E$ belongs to the medial axis. It follows the idea to consider the following algorithm for simplifying $A K^{2}$.

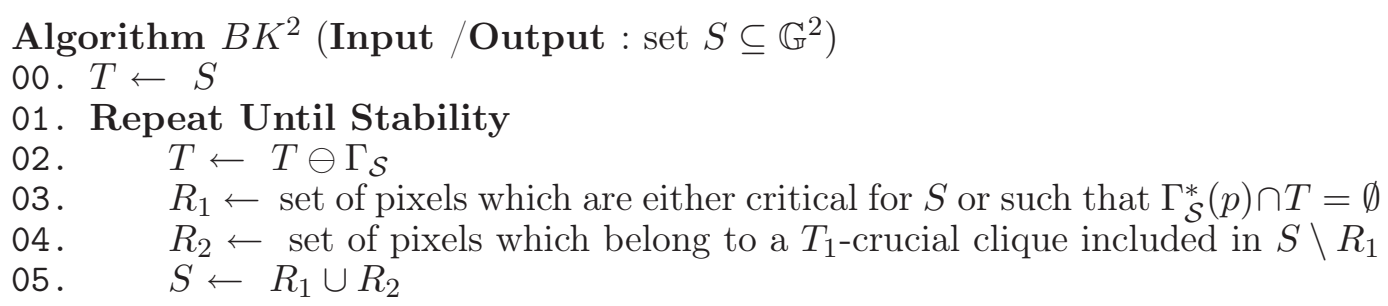

At step 04 of algorithm $B K^{2}$, a pixel in $S \backslash R_{1}$ (a candidate for deletion) cannot belong to the medial axis of $S$. Thus, the skeleton $B K^{2}(S)$ obtained by $B K^{2}$ necessarily contains the medial axis of $S$. Furthermore, we are in the conditions of Prop. 36, this ensures the topological soundness of the algorithm. In Fig. 21, we see that only few pixels are deleted by $A K^{2}$ but not by $B K^{2}$.

\section{Minimal $\mathcal{K}$-skeleton containing the medial axis}

The framework of critical kernels may also be used to design non-symmetric parallel thinning algorithms. We propose here such an algorithm the result of 


$$
\begin{array}{l|c|c|c|c|c|}
\hline \multicolumn{1}{c|}{} & \mathrm{A} & \mathrm{A} \\
\hline & \mathrm{p} & \mathbf{P} \\
\hline N_{1} & \mathrm{~B} & \mathrm{~B} \\
\hline
\end{array}
$$

Fig. 14. Non-symmetric masks. No $\pi / 2$ rotations are considered. The labels $p$ and $P$ indicate pixels that must belong to the set $P$ which is a set composed of simple pixels; the label $p$ (lower case) indicates the position of the pixel which is currently examined. At least one of the pixels marked A and at least one of the pixels marked B must be in $S$.

which includes the medial axis. For that purpose, we consider the following asymmetric variants of the mask $C$ for $T_{1}$-crucial cliques.

Let $S \subseteq \mathbb{G}^{2}$, and let $P$ be a set composed of simple pixels of $S$. We say that a pixel $p$ in $P$ belongs to a $N$-crucial clique included in $P$ if $p$ matches one of the masks $N_{1}, N_{2}$ which are defined Fig. 14 .

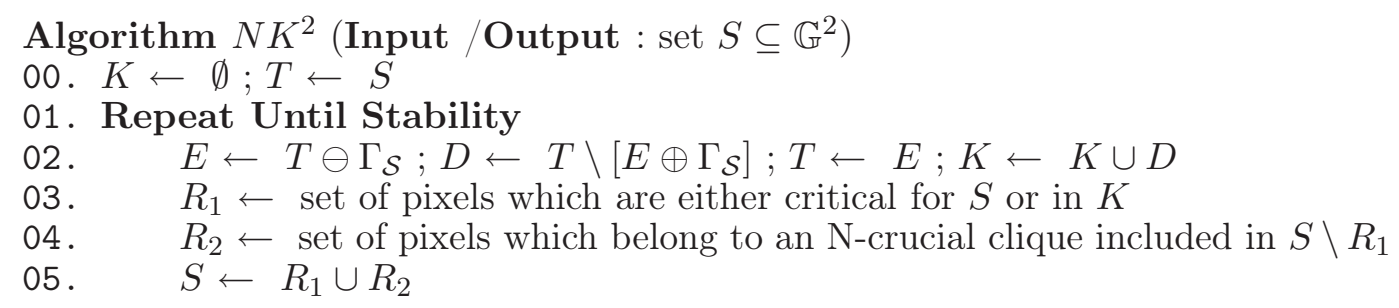

Again we are in the conditions of Prop. 36 , thus $T_{0}$-crucial cliques are preserved by $N K^{2}$. Furthermore, by the very definition of the masks $N_{1}$ and $N_{2}$, at each step 04 of the algorithm, each crucial $T_{1}$-clique included in $S \backslash R_{1}$ contains a pixel which is in $R_{2}$. This ensures the topological soundness of $N K^{2}$. Let us denote by $N K^{2}(S)$ the result of $N K^{2}$ from the input $S$. By construction, $N K^{2}(S)$ contains the medial axis of $S$. Furthermore we have the following property of minimality.

Proposition 38. Let $S \subseteq \mathbb{G}^{2}$. Any pixel in $N K^{2}(S)$ which is not in the medial axis of $S$ is not simple for $N K^{2}(S)$.

Proof: Suppose there are simple pixels in $N K^{2}(S)$ which do not belong to the medial axis of $S$, we denote by $U$ the set composed of all these pixels. It may be seen that each pixel in $U$ matches one of the two masks $N_{1}, N_{2}$ (otherwise such a pixel would have been removed). Let $p$ be one pixel in $U$ which is the "most at the east". Let $q$ be the pixel in $U$ which is the "most at the north of $p$ " and on the same vertical line as $p$. It may be seen that $q$ cannot match one of the masks $N_{1}, N_{2}$, a contradiction.

An example of the result given by the algorithm is shown Fig. 15 (a). See also Fig. 21 for comparisons, in particular with Jang and Chin's algorithm [19] which, like $N K^{2}$, preserves the medial axis and is asymmetric. 


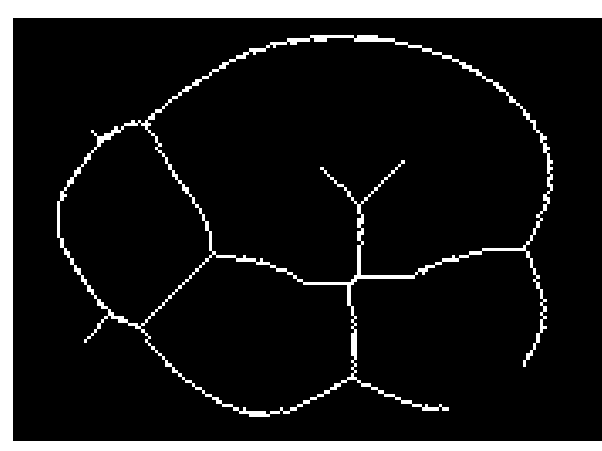

(a)

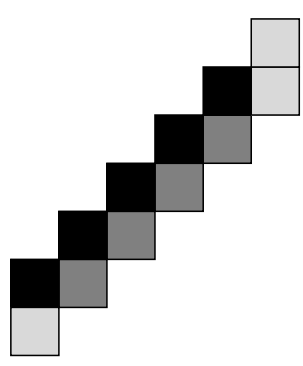

(b)

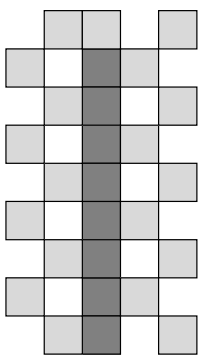

(c)

Fig. 15. (a) The non-symmetric skeleton $N K^{2}(S)$ of the object $S$ depicted Fig. 13, (b) and (c) two configurations where pixels which match $N_{1}$ (resp. $N_{2}$ ) are depicted in black (resp. dark grey), other pixels of the object are in light grey.

The proof of Prop. 38 indicates that there may be the possibility to have, with $N K^{2}$, a "propagation effect" for deleting a simple point. In Fig. 15 (b), all pixels but the extremities of the ribbon are preserved by $N_{1}$ and $N_{2}$. In Fig. 15 (c), the pixel in dark grey at the bottom can be deleted only after all pixels above it are deleted one by one. In fact, such configurations are very likely to contain points of the medial axis, even if they appear after several thinning steps. These points of the medial axis prevent the propagation effect. Algorithm $N K_{2}$ has been tested on 139 binary images. If we denote by $n$ the radius of the largest ball included in an object, the number of iterations was precisely $n$ for 113 images, $n+1$ for 20 images, $n+2$ for 6 images, the radius of each object being between 25 and 110 pixels.

As for $A K^{2}$, we may consider the following simplification of $N K^{2}$. The result of $O K^{2}$ contains the medial axis and also few points which are simple and which do not belong to the medial axis, see Fig. 21.

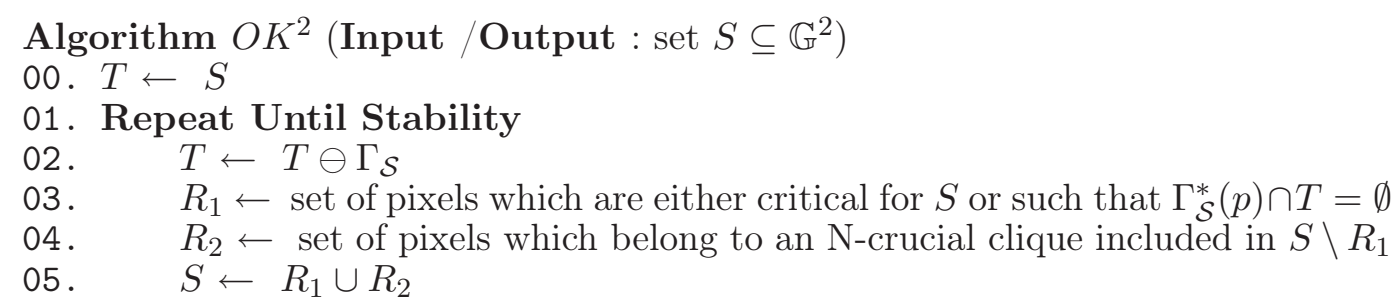

\section{$12 \mathcal{K}$-skeletons of $2 \mathrm{D}$ objects in $3 \mathrm{D}$ grids}

We consider in this section objects which are pure 2-complexes in $\mathbb{F}_{2}^{3}$ as well as objects which are composed of surfels of $\mathbb{F}_{2}^{3}$, i.e., 2-faces of $\mathbb{F}_{2}^{3}$. 
In Sec. 5, we were able to characterize M-critical 1-faces and 0-faces in $\mathbb{F}_{2}^{2}$ directly from the status of the neighboring 2 -faces, opening the way for the simple expression of the masks and algorithms in Sec. 6 and Sec. 7. Although a little less simple, such a characterization is also possible in $\mathbb{F}_{2}^{3}$ for 1 -faces.

Lemma 39. Let $X \sqsubseteq \mathbb{F}_{2}^{3}$, and let $f$ be a 1-face of $X$. Without loss of generality (up to $\pi / 2$ rotations) let us assume that the neighborhood of $f$ is depicted by Fig. 16. The face $f$ is $M$-critical for $X$ if and only if:

i) at least two faces $a_{i}$ are in $X$; and

ii) the faces $a_{i}$ which are in $X$ are all simple for $X$; and

iii) either all the faces $g_{i}$ and $h_{i}$ are not in $X$; or $\left[\left\{g_{0}, \ldots, g_{7}\right\} \cap X \neq \emptyset\right.$ and $\left.\left\{h_{0}, \ldots, h_{7}\right\} \cap X \neq \emptyset\right]$.

Proof: Suppose that $f$ is M-critical for $X$. Since $f$ has to be essential, at least two of the $a_{i}$ 's are in $X$, hence i). By the very definition of an M-critical face, we must also have ii). Since $f$ is critical, the 0 -faces $c$ and $e$ are either both essential or both non-essential for $X$, hence iii). The proof of the converse implication is straightforward.

The case of 0 -faces is much more complex. Take the 0 -face $e$ in Fig. 16. It can be seen that its status depends not only on the one of the 2-faces $h_{i}$ and $a_{i}$ (twelve faces), but also on the status of the six neighboring 0-faces like $c$, and thus on the status of the $6 \times 8=48$ 2-faces like the $g_{i}$ 's. Furthermore, the position of the $g_{i}$ 's which must belong to $X$ in order to determine the status of the 0 -face $c$ depends on the number and position of the $a_{i}$ 's which belong to $X$. Of course, the same is true for the similar groups of 2 -faces surrounding the five other 0 -faces like $c$. In conclusion, a simple combinatorial characterization of M-critical 0 -faces in pure 2-complexes in $\mathbb{F}_{2}^{3}$ cannot be proposed. Fortunately, such a characterization is not mandatory to implement parallel thinning operators based on crucial kernels.

We denote by $\mathbb{G}_{2}^{3}$ the set composed of all 2-faces of $\mathbb{F}_{2}^{3}$. A 2 -face of $\mathbb{G}_{2}^{3}$ is also called a surfel. In the sequel, we consider only finite subsets of $\mathbb{G}_{2}^{3}$.

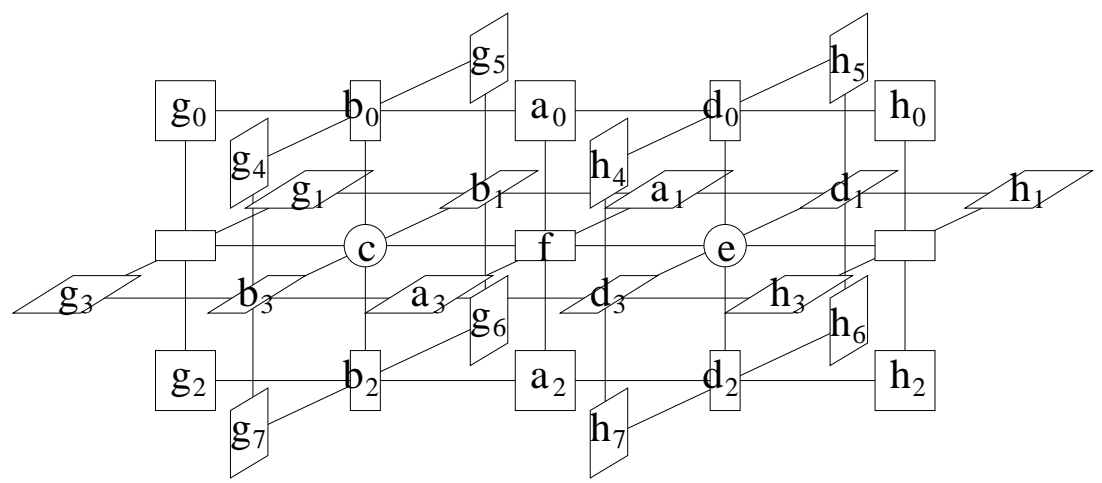

Fig. 16. Naming conventions for Lemma 39. 


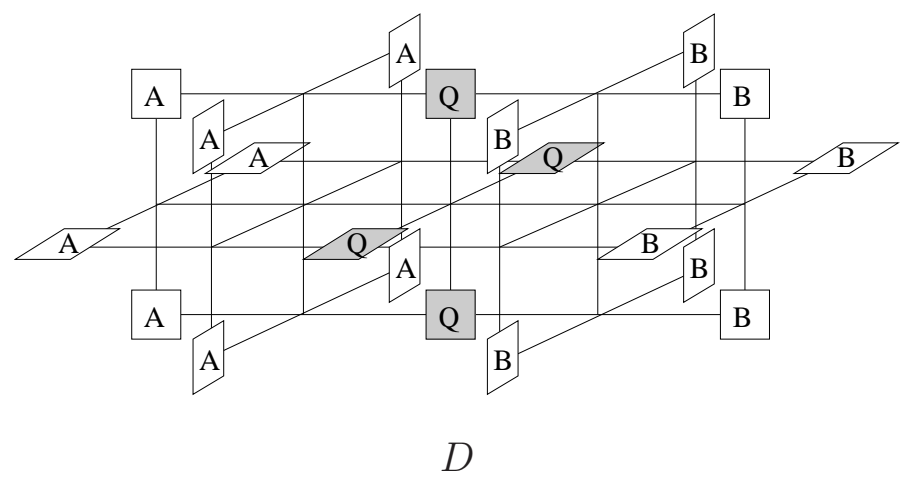

Fig. 17. Pattern and masks for $T_{1}$-crucial surfels. The 3 masks corresponding to this pattern are obtained by applying any series of $\pi / 2$ rotations. The label $Q$ indicates surfels that must either be in $P$ or in the complement of $S$; at least two surfels labeled $Q$ must be in $P$. At least one of the surfels marked $A$ and at least one of the surfels marked $B$ must be in $S$. If one of these masks matches the sets $S, P$, then all the surfels of $P$ which correspond to a label $Q$ are recorded as "matched".

As for the square grid, definitions of facets of $\mathbb{F}_{2}^{3}$ have their counterparts in $\mathbb{G}_{2}^{3}$. For example, if $S \subseteq \mathbb{G}_{2}^{3}$ and $p \in S$, we say that the surfel $p$ is simple for $S$ if $p$ is simple for $S^{-}$. Border, interior, $\left(T_{k^{-}}\right)$critical, and $\left(T_{k^{-}}\right)$crucial surfels are defined in the same manner.

The following property is a direct consequence of Lemma 39. The definition of the pattern $D$ is given Fig. 17 .

Proposition 40. Let $S \subseteq \mathbb{G}_{2}^{3}$, and $p \in S$. Let $P$ be a set composed of simple surfels of $S$. The surfel $p$ belongs to a $T_{1}$-crucial clique included in $P$ if and only if $p$ is matched by the pattern $D$.

The following algorithm computes a minimal $\mathcal{K}$-skeleton, it has exactly the same structure as algorithm $M K^{2}$ for a square grid, but here, the checking of $T_{1}$-crucial elements is made with the mask $D$.

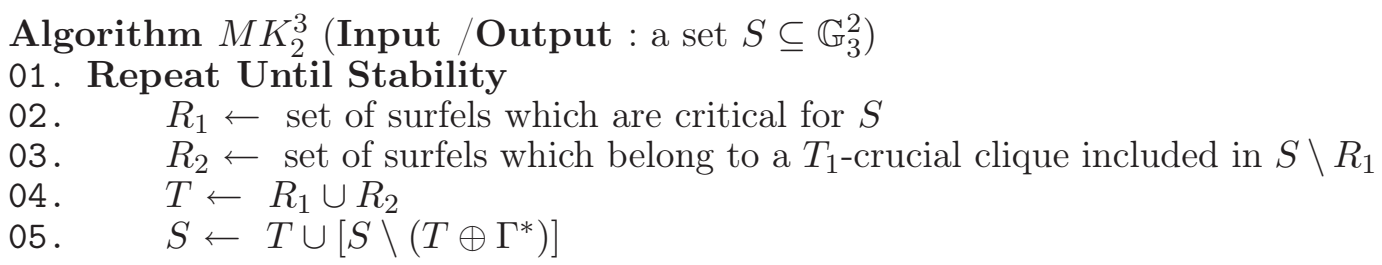

For verifying the topological soundness of the algorithm, we have to prove the analogue of Prop. 27 in $\mathbb{G}_{2}^{3}$. The interested reader may check that this may be done by following exactly all the steps of the proof of Prop. 27, and by establishing the analogue of Prop. 25 in $\mathbb{G}_{2}^{3}$.

An example of a minimal $\mathcal{K}$-skeleton is given Fig. 18. As far as we know, $M K_{2}^{3}$ is the first algorithm for a minimal symmetric skeleton for an object made of 

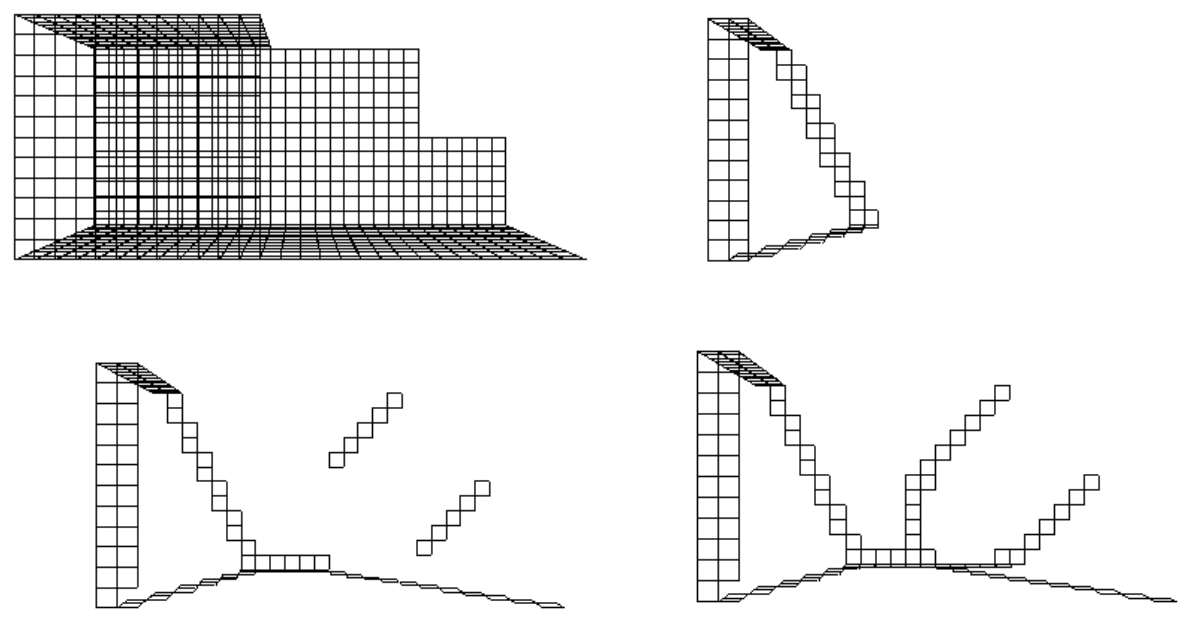

Fig. 18. Top left: A set of surfels $S$ in $\mathbb{F}_{2}^{3}$. Top right: The minimal $\mathcal{K}$-skeleton of $S$. Bottom left: The topological axis of $S$. Bottom right: The result of algorithm $B K_{2}^{3}$.

surfels.

In fact, all algorithms proposed in the last sections may be transposed to $\mathbb{G}_{2}^{3}$. We give here another example, which is a counterpart of algorithm $B K^{2}$.

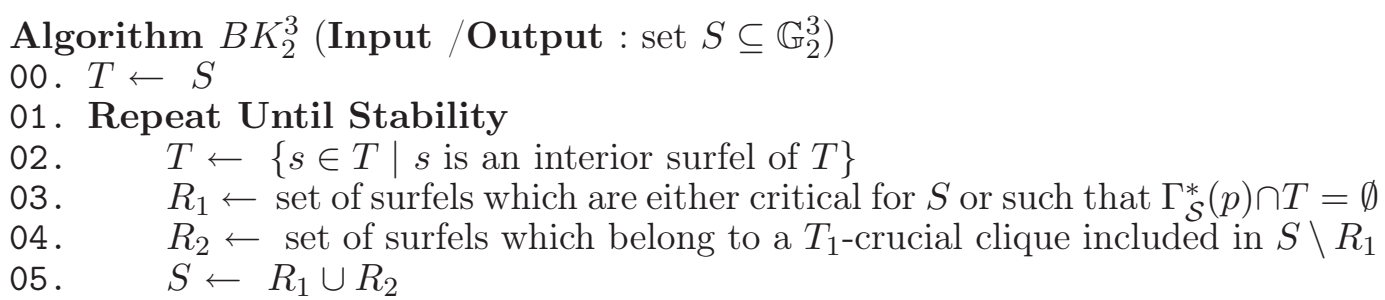

It may be seen that $B K_{2}^{3}(S)$, the result obtained by $B K_{2}^{3}$ from the input $S$, contains the topological axis of $S$. The topological soundness may be proved by establishing the equivalent of Prop. 36 in $\mathbb{G}_{2}^{3}$.

An example of a skeleton obtained with $B K_{2}^{3}$ is given Fig. 18. To our best knowledge, there is only one other algorithm for symmetric curvilinear skeletons of $2 \mathrm{D}$ objects in 3D spaces which is the one given in J. Burguet and R. Malgouyres [7]. This algorithm is based on the technic of P-simple points, see section 14. The 2D objects which are considered are the sets of surfels which constitute the boundary of 3D objects, or subsets of such boundaries. In this context, surfels which share a point or an interval are not necessarily considered as adjacent which makes a difference with the notion of adjacency used in this section. Another difference is that our algorithm always produces a skeleton which contains the topological axis of the original object. 


\section{Checking the topological soundness of thinning algorithms}

Using the framework of critical kernels, it is possible to check, in a concise way, the topological soundness of thinning algorithms. In order to illustrate this fact, we give three proofs of properties relative to three thinning schemes on which quite many thinning algorithms are based. The topological soundness of these schemes has already been established, but we will see that, using the results of this paper, only very simple arguments are needed, in fact these proofs consist in only few lines.

The first thinning scheme is the famous one proposed by A. Rosenfeld [35] which consists in a directional strategy for removing simple pixels in parallel. Simple pixels are classified according to the four directions north, south, east, west. At a given step, all simple and non-end pixels of a given direction are deleted in parallel. The four directions are successively considered.

Let $S \subseteq \mathbb{G}^{2}$, and let $p \in S$. We say that $p$ is a north border pixel (for $S$ ) if the pixel which is strongly adjacent to $p$ and at the north of $p$ does not belong to $S$.

Proposition 41. Let $S \subseteq \mathbb{G}^{2}$, let $P$ be a set of pixels which are both simple and north border pixels for $S$, we set $K=S \backslash P$. Then, any pixel $p$ in $P$ is not $T_{1}$-crucial for $\langle S, K\rangle$. Furthermore, if $p$ is $T_{0}$-crucial for $\langle S, K\rangle$, then $p$ matches either $C_{1}$ or $C_{2}$.

Proof: Let us denote by $C_{a}$ the mask $C$ for $T_{1}$-crucial pixels as it appears Fig. 7 , and by $C_{b}$ its $\pi / 2$ rotation. By the conditions imposed on the A's and B's of mask $C_{a}$, there is, in $C_{a}$, at least one pixel labeled $P$ which is not a north border pixel. The same is true for $C_{b}$, regardless of the A's and B's. Thus a pixel in $P$ cannot match $C_{a}$ or $C_{b}$. By examination of the masks $C_{1}, \ldots, C_{4}$ for $T_{0}$-crucial pixels, we see that a pixel $p$ which is $T_{0}$-crucial for $\langle S, K\rangle$ cannot match $C_{3}$ or $C_{4}$.

Since configurations $C_{1}$ and $C_{2}$ necessarily correspond to end pixels, Prop. 41 proves the soundness of the above thinning scheme.

The second thinning scheme has been proposed by R. Hall. [15]

Let $S \subseteq \mathbb{G}^{2}$, and let $p \in S$. We say that $p$ is a strongly simple pixel for $S$, if $p$ is simple and if $\Gamma^{*}(p) \cap S$ is strongly connected.

The definition of the masks $H_{1}$ and $H_{2}$ considered by R. Hall are given Fig. 19 . Observe that the pixels labeled $H$ are necessarily strongly simple. Thus, these masks may be applied directly on $S$, no preliminary extraction of the set $P$ (or $K$ ) is necessary.

Proposition 42. Let $S \subseteq \mathbb{G}^{2}$, let $P$ be a set of strongly simple pixels for $S$, 


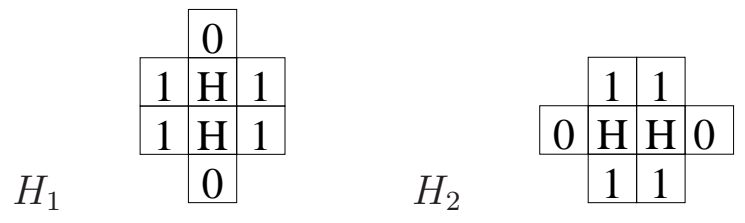

Fig. 19. Masks for $T_{1}$-crucial pixels based on strongly simple pixels. The labels 1 and $H$ (resp. 0) indicate pixels that must belong to the set $S$ (resp. to the complement of $S$ ). If one of these masks matches $S$, then all the pixels with a label $H$ in the mask are recorded as "matched".

and let $K=S \backslash P$. A pixel $p$ is $T_{1}$-crucial for $\langle S, K\rangle$ if and only if $p$ matches the mask $\mathrm{H}_{1}$ or $\mathrm{H}_{2}$.

Proof: If the pixels labeled $P$ in the mask $C$ for $T_{1}$ crucial pixels (see Fig. 7) are strongly simple, then we see that all the A's and all the B's must belong to $S$. This implies that the two pixels on the left and on the right of the $P$ 's in the mask $C$ do not belong to $S$. We thus obtain the masks $H_{1}$ and $H_{2}$.

The third thinning scheme is the one proposed by U. Eckhardt and G. Maderlechner [11]. It consists in considering for deletion a special type of pixels which are not residual (See Def. 32). These pixels may be removed in parallel without any restriction.

Let $S \subseteq \mathbb{G}^{2}$, and let $p \in S$. We say that $p$ is a perfect pixel for $S$, if $p$ is strongly adjacent to an interior pixel $q$ and if the pixel which is at the opposite of $q$ is not in $S$.

Proposition 43. Let $S \subseteq \mathbb{G}^{2}$, let $P$ be a set of pixels which are both simple and perfect for $S$, and let $K=S \backslash P$. Then, any pixel $p$ in $P$ is not crucial for $\langle S, K\rangle$.

Proof: A pixel $p$ in $P$ cannot match the masks $C_{1}, \ldots, C_{4}$ for $T_{0}$-crucial pixels (see Fig. 7). On the other hand, if one of the two pixels labeled $P$ in the mask $C$ is perfect, we easily see that, since the conditions imposed on the A's and B's, the other one cannot be perfect.

In fact, an exhaustive checking of an algorithm given by a set of masks can be realized with the help of a computer. From Def. 15 and Def. 16, an algorithm which does never remove in a single step any critical pixel nor any crucial clique, always produces a crucial retraction of the original object.

Let $X \subseteq \mathbb{G}^{2}$, let $A(X)$ denote the result of one step of a parallel thinning algorithm $A$ on the input $X$. We suppose furthermore that the fact that a pixel $p$ belongs to $A(X)$ or not depends only on the set $X \cap \Gamma^{2}(p)$, where $\Gamma^{2}(p)=\Gamma(\Gamma(p))$. To verify that algorithm $A$ does never delete, e.g. a $T_{0^{-}}$ crucial clique composed of four pixels (mask $C_{4}$ ), it is sufficient to consider a subset $c=\left\{p_{1}, p_{2}, p_{3}, p_{4}\right\}$ of $\mathbb{G}^{2}$ matching $C_{4}$, to generate all the subsets $X$ of 
$\cup_{i=1}^{i=4} \Gamma^{2}\left(p_{i}\right)$ such that $c$ is indeed a crucial clique for $X$, and to check that for any such $X$ the result $A(X)$ contains at least one of the $p_{i}$ 's. These subsets are only 1,048, 576 and can be produced without generating all the $2^{36}$ subsets of $\cup_{i=1}^{i=4} \Gamma^{2}\left(p_{i}\right)$. The same can be done for the other patterns $C, C_{1}, C_{2}, C_{3}$ and all their rotations, as well as for testing that $A$ does preserve critical pixels.

It should be noted that similar computerized tests have already been proposed by R. Hall [16], C.M. Ma [28] for 2D, based on the notion of minimal nonsimple sets [33], and by C.M. Ma [28] for 3D.

We did this work for fifteen parallel thinning algorithms proposed in the literature.

The algorithms proposed by T. Pavlidis in 1981 [31,32], by R.T. Chin, H.K. Wan, D.L. Stover and R.D. Iverson in 1987 [8], by C.M. Holt, A. Stewart, M. Clint and R.D. Perrott in 1987 [17], by R.W. Hall in 1989 [15], by Z. Guo and R.W. Hall in 1992 [14] (3 variants), by B.K. Jang and R.T. Chin in 1993 [19], by U. Eckhardt and G. Maderlechner in 1993 [11], and by T. Bernard and A. Manzanera in 1999 [2] all produce a crucial retraction after a single step of execution. In other words, they all "preserve topology".

On the other hand, the algorithms proposed by D. Rutovitz in 1966 [39], by Y.Y. Zhang and P.S.P. Wang in 1988 [43], by R.Y. Wu and W.H. Tsai in 1992 [42], by B.K. Jang and R.T. Chin in 1992 [18], and by S.S.O. Choy, C.S.T. Choy and W.C. Siu in 1995 [9] do not produce a crucial retraction after a single step of execution. After a quick examination of some configurations detected by our verification program, we found that none of these algorithms does guarantee topology preservation.

A more detailed analysis can be found in [10], where all algorithms are precisely described and counter-examples are shown and discussed.

\section{Minimal non-simple sets and P-simple points}

C. Ronse introduced the minimal non simple sets [33] to propose some conditions under which simple points can be removed in parallel while preserving topology. This leads to verification methods for the topological soundness of 2D thinning algorithms [33,16], 3D thinning algorithms [20,21,29], the 4D case has even been considered in $[12,24,23]$.

Let $S \subseteq \mathbb{G}^{2}$. A sequence $\left\langle p_{0}, \ldots, p_{l}\right\rangle$ is said to be a simple sequence for $S$ if $p_{0}$ is simple for $S$, and if, for any $i=1, \ldots, l, p_{i}$ is simple for the set $S \backslash\left\{p_{j} \mid 0 \leq\right.$ $j<i$ \}. A set $D \subseteq S$ is said to be simple for $S$ if $D$ is empty, or if the pixels 
of $D$ can be ordered as a simple sequence for $S$.

Let $S \subseteq \mathbb{G}^{2}$. A set $M \subseteq S$ is minimal non-simple for $S$ if it is not simple for $S$ and if all its proper subsets are simple.

Observe that if $p$ is not simple for $S$, then $\{p\}$ is a minimal non-simple set.

The verification method with minimal non-simple sets consists in checking that a given algorithm never deletes such sets. We have the following property $[33,16,21]$, we present the formulation proposed in [21].

We say that a subset $M \subseteq \mathbb{G}^{2}$ is small if every two pixels of $M$ are adjacent to each other.

Let $S \subseteq \mathbb{G}^{2}$. Let $M$ be a subset of $S$ which contains only pixels which is are simple for $S$. The set $M \subseteq S$ is minimal non-simple for $S$ if and only if:

i) $M$ is composed of two pixels which are strongly adjacent, such that each pixel of $M$ is not simple for the set obtained from $S$ by deleting the other pixel of $M$; or

ii) $M$ is a small connected component of $S$.

It may be easily seen, by checking the masks $C, C_{1}, \ldots, C_{4}$, that we have the following.

Proposition 44. Let $S \subseteq \mathbb{G}^{2}$. A set $M \subseteq S$ is minimal non-simple for $S$ if and only if:

i) $M$ is composed of a single pixel which is critical for $S$; or

ii) $M$ is a $T_{1}$-crucial clique; or

iii) $M$ is a $T_{0}$-crucial clique.

Thus, critical kernels which are settled in the framework of abstract complexes allow to derive the notion of a minimal non-simple set proposed in the context of digital topology. As illustrated in the preceding section, critical kernels may be used for the verification of algorithms. They can also be used for designing, in an easy way, thinning algorithms such as the ones presented in this paper. In fact, the very definition of a critical kernel may be seen as an algorithm for thinning objects (see Fig. 4).

For the 3D case, also in the framework of digital topology, one of the authors introduced the notion of P-simple points [3].

Let $S \subseteq \mathbb{G}^{2}$, and let $C$ be a subset of $S$. A pixel $p \in C$ is said to be $P$-simple for $\langle S, C\rangle$ if $p$ is simple for all sets $S \backslash T$, such that $T \subseteq C \backslash\{p\}$.

From the conditions proposed for the 3D case in [3], we may verify the following property. 
Proposition 45. Let $S \subseteq \mathbb{G}^{2}$, let $C$ be a subset of $S$, and let $p$ be a simple pixel in $C$. We set $R=S \backslash C$. The pixel $p$ is P-simple for $\langle S, C\rangle$ if and only if:

i) $\Gamma^{*}(p) \cap R$ is non-empty and connected; and

ii) each $q$ in $\Gamma^{*}(p) \cap C$ is adjacent to $\Gamma^{*}(p) \cap R$.

The notion of P-simple point may be used as a verification method, and also as a general methodology to design parallel thinning algorithms $[26,27,7,4]$. For that purpose, it is sufficient to define a subset $C$ of $S$ which contains candidates for deletion, and then to remove in parallel all points which are $\mathrm{P}$-simple for $\langle S, C\rangle$. The following will show the deep link between $\mathrm{P}$-simple points and crucial pixels.

Let $X \preceq \mathbb{F}_{2}^{n}$. Let $C$ be a set of faces which are simple for $X$, we set $K=S \backslash C$. Let $f \in C$. We say that $f$ is weakly crucial for $\langle X, K\rangle$, if $f$ contains a face $g$ which is critical for $X$ and if all 2-faces containing $g$ are in $C$.

From Def. 15 and 18, a face $f$ is crucial for $\langle X, K\rangle$, if $f$ contains a face $g$ which is M-critical for $X$ and if all 2-faces containing $g$ are in $C$. Thus a face which is crucial for $\langle X, K\rangle$ is necessarily weakly crucial for $\langle X, K\rangle$. The interested reader may check the following.

Proposition 46. Let $S \subseteq \mathbb{G}^{2}$, let $C$ be a subset of $S, K=S \backslash C$, and let $p$ be a simple pixel in $C$. The pixel $p$ is P-simple for $\langle S, C\rangle$ if and only if $p$ is not weakly crucial for $\langle S, K\rangle$.

Thus, any pixel which is $\mathrm{P}$-simple for $\langle S, C\rangle$ is not crucial for $\langle S, K\rangle$. The converse is not true. For example, if we consider a set $S$ which consists in a two-pixels width ribbon, and if we set $C=S$, it may be seen that the four pixels at the extremities of the ribbon are not crucial for $\langle S, K\rangle$ but not Psimple for $\langle S, C\rangle$. Indeed, it is possible to remove more pixels with a thinning scheme which deletes simple pixels which are not crucial than with one which deletes P-simple pixels.

In fact, despite the appearance, it is possible to check only with the notion of P-simple points whether the result of one step of a given algorithm is a crucial retraction or not. Since every critical face is included in an M-critical face, by Prop. 46, we have the following.

Proposition 47. Let $S \subseteq \mathbb{G}^{2}$, let $T$ be a subset of $S$. The set $T$ is a crucial retraction of $S$ if and only if each $p$ in $S \backslash T$ is P-simple for $\langle S, S \backslash T\rangle$. 


\section{Conclusion}

Based on the framework of critical kernels [5], we studied the case of 2D structures in 2D and 3D grids. The salient outcomes of this article are the following:

- the definition and some characterizations of crucial faces, allowing for fast and simple implementations,

- the definition and an algorithm for a minimal symmetric skeleton $\left(M K^{2}\right)$,

- a symmetrical thinning algorithm based on ends $\left(E K^{2}\right)$,

- a symmetrical thinning algorithm based on residues $\left(R K^{2}\right)$,

- the introduction of the topological axis, which generalizes the medial axis,

- two parallel algorithms for a symmetric skeleton which contains the medial axis $\left(A K^{2}\right.$ and $\left.B K^{2}\right)$,

- a parallel algorithm for a minimal skeleton which contains the medial axis $\left(N K^{2}\right)$, and also a "sub-optimal" variant $\left(O K^{2}\right)$,

- a parallel algorithm for a minimal symmetric skeleton of an object made of surfels $\left(M K_{2}^{3}\right)$,

- a parallel algorithm for a symmetric skeleton, which contains the topological axis of an object made of surfels $\left(B K_{2}^{3}\right)$.

As far as we know, all the above algorithms have no equivalent.

The methodology presented in this paper has been extended to the important case of parallel thinning of 3D objects [6]. In future works, we will study the case of general skeletons (i.e., which are not necessarily principal subcomplexes), the computation of Euclidean skeletons, and the link between critical kernels, minimal non-simple sets and P-simple points for the 3D- and 4D-cases.

\section{References}

[1] C. Arcelli, L.P. Cordella, and S. Levialdi. Parallel thinning of binary pictures. Electronic Letters, 11(7):148-149, 1975.

[2] T.M. Bernard and A. Manzanera. Improved low complexity fully parallel thinning algorithm. In Proceedings 10th International Conference on Image Analysis and Processing (ICIAP'99), 1999.

[3] G. Bertrand. On P-simple points. Comptes Rendus de l'Académie des Sciences, Série Math., I(321):1077-1084, 1995.

[4] G. Bertrand. Sufficient conditions for 3D parallel thinning algorithms. In SPIE Vision Geometry IV, volume 2573, pages 52-60, 1995. 
[5] G. Bertrand. On critical kernels. Internal Report. Université de Marne-la-Vallée, IGM2005-05, 2005. Also submitted for publication.

[6] G. Bertrand and M. Couprie. A new 3D parallel thinning scheme based on critical kernels. Lecture Notes in Computer Science, 4245:580-591, 2006.

[7] J. Burguet and R. Malgouyres. Strong thinning and polyhedric approximation of the surface of a voxel object. Discrete Applied Mathematics, 125:93-114, 2003.

[8] R.T. Chin, H.K. Wan, D.L. Stover, and R.D. Iverson. A one-pass thinning algorithm and its parallel implementation. Computer Vision, Graphics, and Image Processing, 40(1):30-40, October 1987.

[9] S.S.O. Choy, C.S.T. Choy, and W.C. Siu. New single-pass algorithm for parallel thinning. Computer Vision and Image Understanding, 62(1):69-77, July 1995.

[10] M. Couprie. Note on fifteen 2D parallel thinning algorithms. Internal Report, Université de Marne-la-Vallée, IGM2006-01, 2005.

[11] U. Eckhardt and G. Maderlechner. Invariant thinning. International Journal of Pattern Recognition and Artificial Intelligence, 7(5):1115-1144, 1993.

[12] C-J. Gau and T.Y. Kong. Minimal non-simple sets in 4D binary pictures. Graphical Models, 65:112-130, 2003.

[13] P. Giblin. Graphs, surfaces and homology. Chapman and Hall, 1981.

[14] Z. Guo and R.W. Hall. Fast fully parallel thinning algorithms. Computer Vision, Graphics, and Image Processing, 55(3):317-328, May 1992.

[15] R.W. Hall. Fast parallel thinning algorithms: Parallel speed and connectivity preservation. Communication of the ACM, 32(1):124-131, January 1989.

[16] R.W. Hall. Tests for connectivity preservation for parallel reduction operators. Topology and its Applications, 46(3):199-217, 1992.

[17] C.M. Holt, A. Stewart, M. Clint, and R.H. Perrott. An improved parallel thinning algorithm. CACM, 30(2):156-160, February 1987.

[18] B.K. Jang and R.T. Chin. One-pass parallel thinning: Analysis, properties, and quantitative evaluation. IEEE Pattern Analysis and Machine Intelligence, 14(11):1129-1140, November 1992.

[19] B.K. Jang and R.T. Chin. Reconstructable parallel thinning. Pattern Recognition and Artificial Intelligence, 7:1145-1181, 1993.

[20] T.Y. Kong. On the problem of determining whether a parallel reduction operator for n-dimensional binary images always preserves topology. In procs. SPIE Vision Geometry II, volume 2060, pages 69-77, 1993.

[21] T.Y. Kong. On topology preservation in 2-D and 3-D thinning. International Journal on Pattern Recognition and Artificial Intelligence, 9:813-844, 1995. 
[22] T.Y. Kong. Topology-preserving deletion of 1's from 2-, 3- and 4-dimensional binary images. In Lecture Notes in Computer Science, volume 1347, pages 3-18, 1997.

[23] T.Y. Kong. Minimal non-simple and minimal non-cosimple sets in binary images on cell complexes. In Lecture Notes in Computer Science, volume 4245, pages 169-188, 2006.

[24] T.Y. Kong and C-J. Gau. Minimal non-simple sets in 4-dimensional binary images with (8-80)-adjacency. In Procs. Int. Workshop on Combinatorial Image Analysis, pages 318-333, 2004.

[25] T.Y. Kong and A. Rosenfeld. Digital topology: introduction and survey. Comp. Vision, Graphics and Image Proc., 48:357-393, 1989.

[26] C. Lohou and G. Bertrand. A 3D 12-subiteration thinning algorithm based on P-simple points. Discrete Applied Mathematics, 139:171-195, 2004.

[27] C. Lohou and G. Bertrand. A 3D 6-subiteration curve thinning algorithm based on P-simple points. Discrete Applied Mathematics, 151:198-228, 2005.

[28] C.M. Ma. Connectivity preserving transformation of digital images: theory and application. PhD thesis, The City University of New-York, 1994.

[29] C.M. Ma. On topology preservation in 3D thinning. CVGIP: Image Understanding, 59(3):328-339, May 1994.

[30] A. Manzanera and T.M. Bernard. Metrical properties of a collection of 2D parallel thinning algorithms. In Electronic Notes on Discrete Mathematics, Proc. 9th IWCIA, volume 12, 2003.

[31] T. Pavlidis. A flexible parallel thinning algorithm. In Proc. IEEE Comput. Soc. Conf. Pattern Recognition, Image Processing, pages 162-167, 1981.

[32] T. Pavlidis. An asynchronous thinning algorithm. Computer Graphics and Image Processing, 20(2):133-157, October 1982.

[33] C. Ronse. Minimal test patterns for connectivity preservation in parallel thinning algorithms for binary digital images. Discrete Applied Mathematics, 21(1):67-79, 1988.

[34] A. Rosenfeld. Connectivity in digital pictures. Journal of the Association for Computer Machinery, 17:146-160, 1970.

[35] A. Rosenfeld. A characterization of parallel thinning algorithms. Information and control, 29(3):286-291, 1975.

[36] A. Rosenfeld. Digital topology. American Mathematical Monthly, 86:621-630, 1979.

[37] A. Rosenfeld and A.C. Kak. Digital picture processing. Academic Press, 1982.

[38] A. Rosenfeld and J.L. Pfaltz. Sequential operations in digital picture processing. Journal of the Association for Computer Machinery, 13:471-494, 1966. 
[39] D. Rutovitz. Pattern recognition. Journal of the Royal Statistical Society, 129:504-530, 1966.

[40] J. Serra. Image analysis and mathematical morphology. Academic Press, 1982.

[41] R. Stefanelli and A. Rosendeld. Some parallel thinning algorithms for digital pictures. Journal of the Association for Computing Machinery, 18(2):255-264, 1971.

[42] R.Y. Wu and W.H. Tsai. A new one-pass parallel thinning algorithm for binary images. Pattern Recognition Letters, 13:715-723, 1992.

[43] Y.Y. Zhang and P.S.P. Wang. A modified parallel thinning algorithm. In International Conference on Pattern Recognition, pages 1023-1025, 1988. 


\section{Appendix A}

This appendix gives the two lemmas which lead to the characterization of end pixels of Prop. 30 .

Lemma 48. Let $X \sqsubseteq \mathbb{F}_{2}^{2}$, let $f$ be a 2-face of $X$ which contains exactly one critical face $m$, and such that $m$ is a 1-face. Without loss of generality (up to $\pi / 2$ rotations), suppose that $m$ is located as $b_{0}$ in Fig. 6(c). Then,

i) $a_{0} \in X$; and

ii) $a_{i} \notin X$, for any $i=1, \ldots, 7$.

Proof: since $b_{0}$ is critical, it must be essential, hence $a_{0} \in X$. The 0 -faces $c_{i}$ $(i=0, \ldots, 3)$ cannot be essential otherwise they would be critical, thus the faces $a_{1}, a_{3}, a_{5}, a_{7}$ are not in $X$. The 1 -face $b_{1}$ is not critical by hypothesis, thus it cannot be essential, otherwise since $c_{0}, c_{1}$ are both non-essential $b_{1}$ would be critical. The same holds for $b_{2}, b_{3}$, thus $a_{2}, a_{4}, a_{6}$ are not in $X$.

Lemma 49. Let $X \sqsubseteq \mathbb{F}_{2}^{2}$, let $f$ be a 2-face of $X$ which contains exactly one critical face $m$, and such that $m$ is a 0-face. Without loss of generality (up to $\pi / 2$ rotations), suppose that $m$ is located as $c_{0}$ in Fig. $6(c)$. Then,

i) $a_{1} \in X$ or $\left\{a_{0}, a_{2}\right\} \subset X$ (or both); and

ii) $a_{i} \notin X$, for any $i=3, \ldots, 7$.

Proof: since $c_{0}$ is critical, it must be essential, thus (since $X$ is a pure 2complex) we must have i). By hypothesis $c_{1}, c_{2}, c_{3}$ are non-critical, thus they are non-essential, and also $b_{2}, b_{3}$ are non-essential (otherwise they would be critical), hence ii). 


\section{Appendix B}

This appendix gives a counter-example for the preservation of the medial axis with residual pixels (algorithm $R K^{2}$ ). In Fig. 20(a) an original object $S \in \mathbb{G}^{2}$ with one hole (the "black square") and the associated complex $X=S^{-}$are depicted. The medial axis of $S$ is highlighted by a bold contour. In (b), the set $P$ of pixels which are simple for $S$ and which are not residues is highlighted by a bold contour. The M-critical faces of $X$ which are $T_{1}$-critical are depicted in gray. We see that the faces $x$ and $y$ constitute a $T_{1}$-crucial clique included in $S \backslash P$. Thus, at the first step of algorithm $R K^{2}$, all the pixels of $P$ but $x$ and $y$ will be deleted. The resulting complex $X_{1}$ after the first step of algorithm $R K^{2}$ is given in (c). We set $S_{1}=X_{1}^{+}$. Again, the set $P_{1}$ of pixels which are simple for $S_{1}$ and which are not residues is highlighted by a bold contour, and the M-critical faces of $X_{1}$ which are $T_{1}$-critical are depicted in gray. There is no $T_{1}$-crucial clique included in $S_{1} \backslash P_{1}$. Thus, at the second step of algorithm $R K^{2}$, all the pixels of $P_{1}$, including the medial axis pixel $z$, will be deleted.

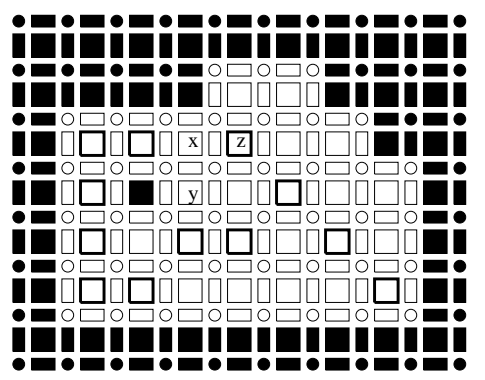

(a)

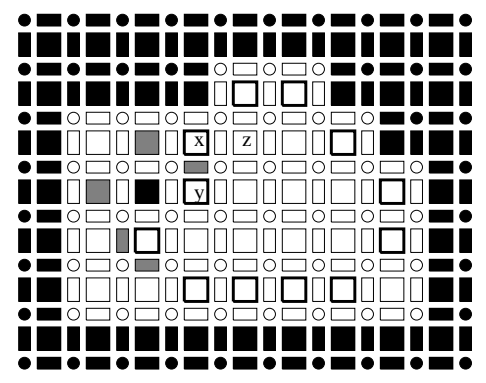

(b)

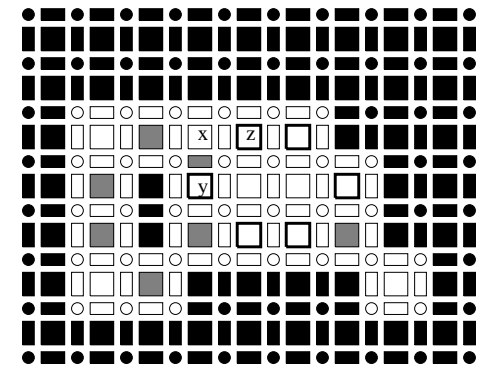

(c)

Fig. 20. A counter-example for the preservation of the medial axis with residual pixels. 


\section{Appendix C}

This appendix gives some experimental results related to the number of points which appear in the skeleton obtained by several parallel thinning algorithms. It should be noted that some algorithms were not designed for containing as much as possible points of the medial axis (such as Bernard and Manzanera's algorithm).

\begin{tabular}{l|c|c||c|c||c|c} 
Algorithm & $N_{1}$ & $A_{1}$ & $N_{2}$ & $A_{2}$ & $N_{3}$ & $A_{3}$ \\
\hline Medial axis (reference) & - & 564 & - & 1359 & - & 2178 \\
Pavlidis 1981 & 847 & 564 & 2829 & 1359 & 4241 & 2172 \\
Chin, Wan et al. 1987 & 544 & 153 & 1572 & 334 & 3057 & 778 \\
Hall 1989 & 591 & 467 & 1773 & 1103 & 3060 & 1557 \\
Guo and Hall 1992 & 560 & 437 & 1664 & 993 & 3149 & 1750 \\
Jang and Chin 1993 & 704 & 564 & 2394 & 1359 & 3787 & 2178 \\
Eckhardt and Maderlechner 1993 & 724 & 564 & 2434 & 1359 & 3895 & 2171 \\
Bernard and Manzanera 1999 & 678 & 534 & 1929 & 1219 & 3528 & 2018 \\
$E K^{2}$ & 869 & 561 & 2966 & 1319 & 4475 & 2086 \\
$R K^{2}$ & 739 & 562 & 2581 & 1330 & 3762 & 2054 \\
$A K^{2}$ & 743 & 564 & 2649 & 1359 & 4065 & 2178 \\
$B K^{2}$ & 744 & 564 & 2680 & 1359 & 4080 & 2178 \\
$N K^{2}$ & 677 & 564 & 2349 & 1359 & 3577 & 2178 \\
$O K^{2}$ & 678 & 564 & 2365 & 1359 & 3667 & 2178
\end{tabular}

Fig. 21. $N_{i}$ : number of pixels in the skeleton. $A_{i}$ : number of pixels of the skeleton which belong to the medial axis. The index $i$ refers to the shape number in Fig. 22.

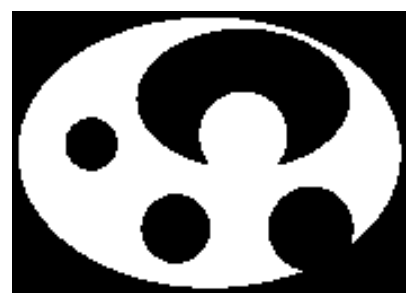

(1)

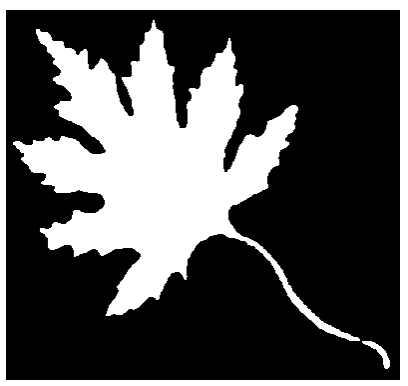

(2)

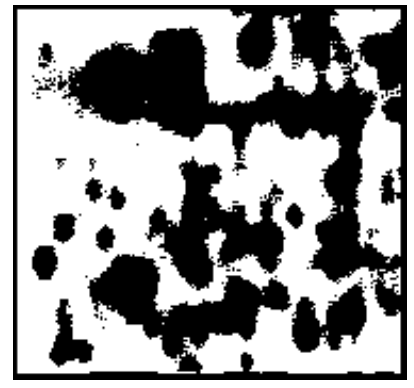

(3)

Fig. 22. Three shapes (in white) for the comparison of thinning algorithms. 